\title{
Representing a cubic graph as the intersection graph of axis-parallel boxes in three dimensions
}

\author{
Abhijin Adiga $^{1}$, L. Sunil Chandran ${ }^{1}$ \\ Department of Computer Science and Automation, Indian Institute of Science, Bangalore-560012, India. \\ email: $\{$ abhijin,sunil\}@csa.iisc.ernet.in
}

\begin{abstract}
We show that every graph of maximum degree 3 can be represented as the intersection graph of axis parallel boxes in three dimensions, that is, every vertex can be mapped to an axis parallel box such that two boxes intersect if and only if their corresponding vertices are adjacent. In fact, we construct a representation in which any two intersecting boxes just touch at their boundaries. Further, this construction can be realized in linear time.
\end{abstract}

Key words: cubic graphs, intersection graphs, axis parallel boxes, boxicity

\section{Introduction}

We will be considering only simple, undirected and finite graphs. Let $\mathcal{F}=\left\{S_{1}, S_{2}, \ldots, S_{n}\right\}$ be a family of sets. An intersection graph associated with $\mathcal{F}$ has $\mathcal{F}$ as the vertex set and two vertices $S_{i}$ and $S_{j}$ are adjacent if and only if $i \neq j$ and $S_{i} \cap S_{j} \neq \emptyset$. It is interesting to study intersection graphs of sets with some restriction, for example, sets which correspond to geometric objects such as intervals, spheres, boxes, axis-parallel lines, etc. Many important graph classes arise out of such restrictions: interval graphs, circular arc graphs, unitdisk graphs and grid-intersection graphs, to name a few. In this paper, we are concerned with intersection graphs of 3-dimensional boxes. A 3-dimensional axis parallel box (3-box in short) is a Cartesian product of 3 closed intervals on the real line. A graph is said to have a 3-box representation if it can be represented as the intersection graph of 3-boxes.

In the literature there are several results on representing a planar graph as the intersection graph of various geometric objects. Among these, the most noted result is the circle packing theorem (also known as the Koebe-Andreev-Thurston theorem) from which it follows that planar graphs are exactly the intersection graphs of closed disks in the plane such that the intersections happen only at the boundaries. In [12, Thomassen gave a similar representation for planar graphs with 3-boxes. He showed that every planar graph has a strict 3-box representation, that is, intersections occur only in the boundaries of the boxes and two boxes which intersect have precisely a 2-box (a rectangle) in common. Very recently, Felsner and Francis [7] strengthened this result by showing that there exists a strict 3-box representation for a planar graph such that each box is an isothetic cube. In [10]12, it was shown that every planar graph has a strict representation using at most two rectangles per vertex. Scheinerman and West [1] showed that every planar graph is an intersection graph of intervals such that each vertex is represented by at most three intervals on the real line.

We consider the question of whether a graph of maximum degree 3 has a 3 -box representation. We note that there exist graphs with maximum degree greater than 3 which do have a 3-box representation. For example, it is easy to show that a $K_{8}$ minus a perfect matching does not have a 3 -box representation 9 . Considering the effort that has gone into discovering geometric representation theorems for planar graphs, it is surprising that no such results are known up to now in the case of cubic graphs. It may be because of the fact that intuitively cubic graphs are farther away from "geometry" compared to planar graphs. In this paper we present the first such theorem (as far as we know) for cubic graphs:

Theorem 1. Every graph of maximum degree 3 has a 3-box representation with the restriction that two boxes can intersect only at their boundaries.

\section{$1.1 k$-box representations and boxicity}

The concept of 3-box representation can be extended to higher dimensions. A $k$-box is a Cartesian product of closed intervals $\left[a_{1}, b_{1}\right] \times\left[a_{2}, b_{2}\right] \times \cdots \times\left[a_{k}, b_{k}\right]$. A graph $G$ has a $k$-box representation if it is the intersection 
graph of a family of $k$-boxes in the $k$-dimensional Euclidean space. The boxicity of $G$ denoted by box $(G)$, is the minimum integer $k$ such that $G$ has a $k$-box representation. Clearly, Theorem 1 can be rephrased as: Every graph with maximum degree 3 has boxicity at most 3 . The best known upper bound for the boxicity of cubic graphs is 10; it follows from the bound box $(G) \leq 2\left\lfloor\frac{\Delta^{2}}{2}\right\rfloor+2$ by Esperet [6], where $\Delta$ is the maximum degree of the graph. In 2, it was conjectured that boxicity of a graph is $O(\Delta)$. However, this was disproved in [1] by showing the existence of graphs with boxicity $\Omega(\Delta \log \Delta)$. Theorem 1 implies that the conjecture is true for $\Delta=3$.

Our result also implies that any problem which is hard for cubic graphs is also hard for graphs with a 3-box representation. We list a few of such problems: crossing number, minimum vertex cover, Hamiltonian cycle, maximum independent set, minimum dominating set and maximum cut.

We give a brief literature survey on boxicity. It was introduced by Roberts in 1969 9. Cozzens [4] showed that computing the boxicity of a graph is NP-hard. This was later strengthened by Yannakakis [13] and finally by Kratochvil [ 8 ] who showed that determining whether boxicity of a graph is at most two itself is NP-complete. Adiga, Bhowmick and Chandran [1] showed that it is hard to approximate the boxicity of even a bipartite graph within $\sqrt{n}$ factor, where $n$ is the order of the graph. In [5], Cozzens and Roberts studied the boxicity of split graphs. Chandran and Sivadasan $[3]$ showed that box $(G) \leq$ tree-width $(G)+2$. Chandran, Francis and Sivadasan [2] proved that box $(G) \leq 2 \chi\left(G^{2}\right)$, where $\chi$ is the chromatic number and $G^{2}$ is the square of the graph.

Boxicity is a direct generalization of the concept of interval graphs. A graph is an interval graph if and only if it can be expressed as the intersection of a family of intervals on the real line. Since a 1-box is an interval, it follows that interval graphs are precisely the class of graphs with boxicity at most 11 . Now we present an alternate characterization of $k$-box representation in terms of interval graphs. This is used more frequently than its geometric definition.

Lemma 1. A graph $G$ has a $k$-box representation if and only if there exist $k$ interval graphs $I_{1}, I_{2}, \ldots, I_{k}$ such that $V\left(I_{i}\right)=V(G), i=1,2, \ldots, k$ and $E\left(I_{1}\right) \cap E\left(I_{2}\right) \cap \cdots \cap E\left(I_{k}\right)=E(G)$.

Our proof of Theorem 1 uses Lemma 1, we construct 3 interval graphs such that the given cubic graph is the intersection of these interval graphs.

We observe that there exist graphs with maximum degree 3 (and hence cubic graphs) with boxicity strictly greater than 2 . For example, Let $G$ be a non-planar cubic graph and $G_{s}$ be the graph obtained by subdividing each edge once. Then, $\operatorname{box}\left(G_{s}\right)>2$. It is an easy exercise to prove this. One way is to show that if $G_{s}$ does have a 2-box representation, then a planar embedding for $G$ can be derived from this box representation, contrary to the initial assumption that $G$ is a non-planar graph. This means that these graphs do not have a 2-box representation, that is, they cannot be expressed as the intersection graphs of rectangles on the plane. The rest of the paper is devoted to the proof of Theorem 1 .

\subsection{Notation}

Let $G$ be a graph. The notation $(x, y) \in E(G)((x, y) \notin E(G))$ means that $x$ is (not) adjacent to $y$ in $G$. For $U \subseteq V(G), G[U]$ denotes the graph induced by $U$ in $G$. The open neighborhood of $U$, denoted by $N(U, G)$ is the set $\{x \in V(G) \backslash U \mid \exists y \in U$ such that $(x, y) \in E(G)\}$. The length of a path is the number of edges in the path. We consider an isolated vertex as a path of length 0. Suppose $G$ and $H$ are graphs defined on the same vertex set. $G \cap H$ denotes the graph with $V(G \cap H)=V(G)(=V(H))$ and $E(G \cap H)=E(G) \cap E(H)$.

Consider a non-empty set $X$ and let $\Pi$ be an ordering of the elements of $X . \bar{\Pi}$ denotes the reverse of $\Pi$, that is, for any $x, y \in X, \bar{\Pi}(x)<\bar{\Pi}(y)$ if and only if $\Pi(x)>\Pi(y)$. Let $A$ and $B$ be disjoint subsets of $X$. The notation $\Pi(A)<\Pi(B)$ implies the following: $\forall a \in A, b \in B, \Pi(a)<\Pi(b)$.

Lemma 2. If every cubic graph has a 3-box representation, then, every graph of maximum degree 3 also has a 3-box representation. The statement holds even when the intersections are restricted to the boundaries of the boxes.

\footnotetext{
${ }^{1}$ The only graph with boxicity 0 is the complete graph.
} 
Proof. Let $H$ be a non-cubic graph with maximum degree 3. We will show that there exists a cubic graph $H^{\prime}$ such that $H$ is an induced subgraph of $H^{\prime}$. Here is one way of constructing $H^{\prime}$ from $H$. Let $D=3|V(H)|-$ $\sum_{v \in V(H)} d(v)$, where $d(v)$ is the degree of $v$. Let $C_{D}$ be a $D$-length cycle such that $V\left(C_{D}\right) \cap V(H)=\varnothing$. We construct $H^{\prime}$ as follows: Let $V\left(H^{\prime}\right)=V(H) \cup V\left(C_{D}\right)$. $H^{\prime}$ contains all the edges contained in $H$ and $C_{D}$ and in addition, each vertex $v \in V(H)$ is made adjacent to $3-d(v)$ unique vertices from $V\left(C_{D}\right)$, where $d(v)$ is the degree of $v$. Clearly, $H^{\prime}$ is cubic and by Theorem 1, has a 3-box representation. Since $H$ is an induced subgraph of $H^{\prime}$, any box representation of $H^{\prime}$ can be converted to a box representation of $H$ by simply retaining only the boxes of vertices belonging to $H$.

In view of Lemma 2, we note that it is enough to prove that a cubic graph has a 3-box representation. Therefore, in our proof of Theorem 1, we will assume that the graph is cubic.

\section{Structural prerequisites}

\subsection{Special cycles and paths}

Definition 1. Special cycle: An induced cycle $C$ is a special cycle if for all $x \in C, C \backslash\{x\}$ is not a subgraph of an induced cycle or path of size $\geq|C|+1$.

Definition 2. Special path: An induced path $P$ is a special path if

1. it is maximal in the sense that it is not a subgraph of an induced cycle or a longer induced path, and

2. for any end point of $P$, say $x, P \backslash\{x\}$ is not a subgraph of an induced cycle of size $\geq|P|$ or an induced path of length $\geq|P|+1$.

Observation 1. Any connected graph with at least 3 vertices contains a special cycle or path.

This is easy to see. Among all sets of vertices which induce cycles or paths in the graph, consider the largest sets. If one of these sets induces a cycle, then, clearly this is a special cycle since there is no larger induced path or cycle in the graph. If none of them induces a cycle, then, each of these sets induces a special path since there is no induced longer path or an induced cycle of the same size in the graph.

\subsection{Partitioning the vertex set of a cubic graph}

Let $G$ be a cubic graph and let $V=V(G)$. We partition $V$ in two stages. In Algorithm 1, we obtain the primary partition: $V=\mathcal{S} \uplus \mathcal{N}_{1} \uplus \mathcal{A}_{1}$. This is followed by a finer partitioning in Algorithm 2 $\mathcal{N}_{1}=\mathcal{R} \uplus \mathcal{N}$ and $\mathcal{A}_{1}=\mathcal{B} \uplus \mathcal{A}$.

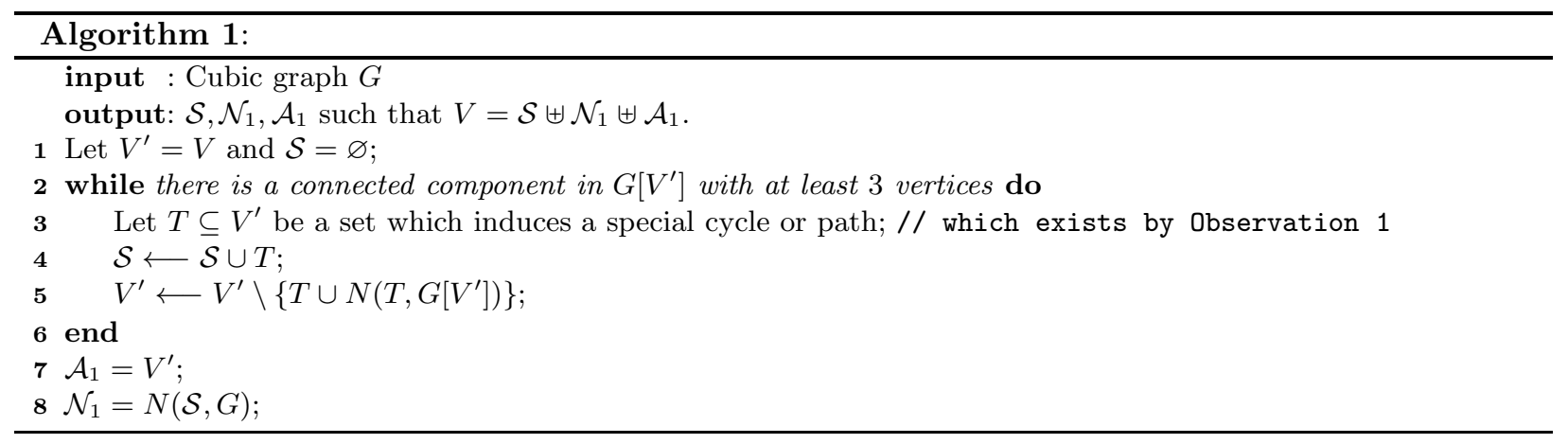

Observation 2. We have some easy observations from Algorithm 1: 
1. $\mathcal{S}$ induces a collection of cycles and paths in $G$.

2. Every vertex in $\mathcal{S}$ has at least one neighbor in $\mathcal{N}_{1}$. Therefore, every vertex in $\mathcal{N}_{1}$ is adjacent to at most two vertices in $\mathcal{A}_{1}$.

3. For any $u \in \mathcal{S}$ and $v \in \mathcal{A}_{1}, u$ and $v$ are not adjacent.

4. $\mathcal{A}_{1}$ induces a collection of isolated vertices and edges in $G$. This observation follows from the fact that $G\left[\mathcal{A}_{1}\right]$ does not contain any special cycle or path and therefore, from Observation 1 , does not contain any component with three or more vertices.

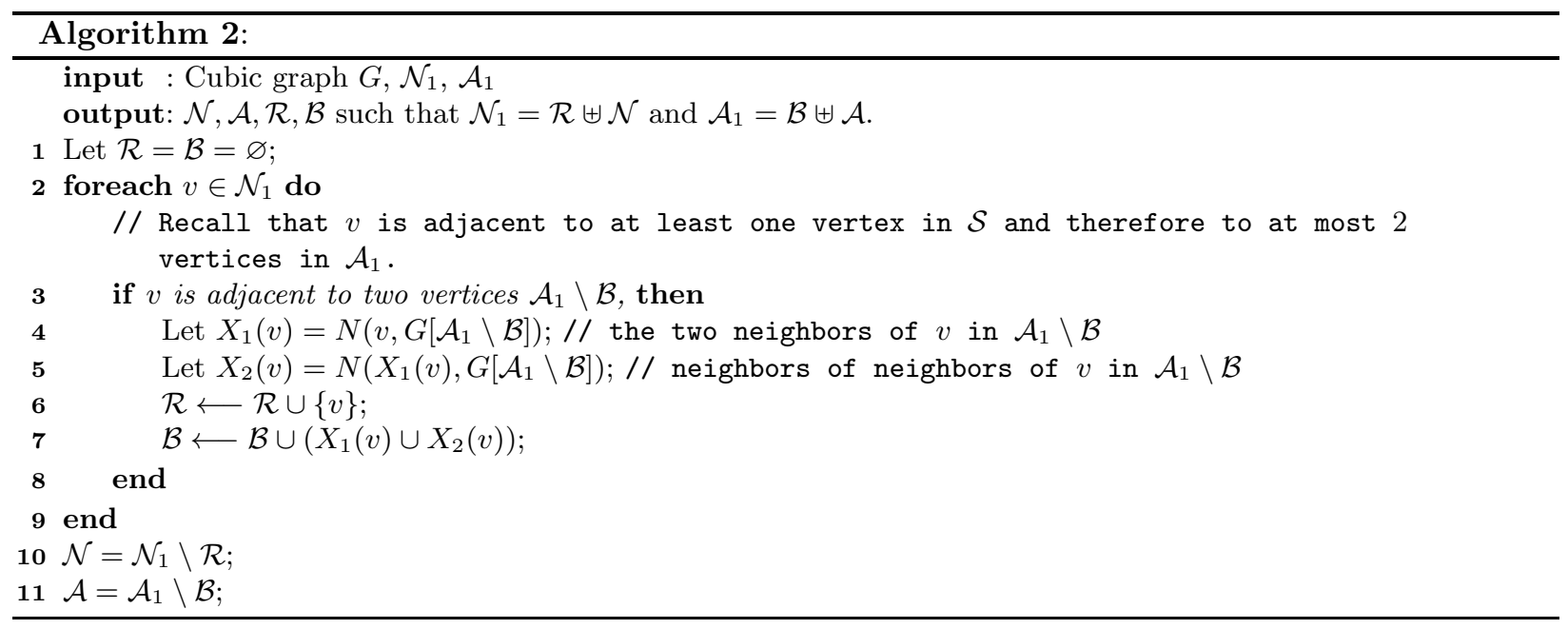

Observation 3. Some observations from Algorithm 2 .

1. Every vertex in $\mathcal{N}$ is adjacent to at most one vertex in $\mathcal{A}$.

2. Every vertex in $\mathcal{R}$ is adjacent to one vertex in $\mathcal{S}$ and two vertices in $\mathcal{B}$. This immediately implies that (a) $\mathcal{R}$ is an independent set and (b) for any $u \in \mathcal{R}$ and $v \in \mathcal{N} \cup \mathcal{A}, u$ and $v$ are not adjacent.

3. Since $\mathcal{B} \subseteq \mathcal{A}_{1}$, for any $u \in \mathcal{B}$ and $v \in \mathcal{S}, u$ and $v$ are not adjacent, by Observation 23.

4. For any $u \in \mathcal{B}$ and $v \in \mathcal{A}, u$ and $v$ are not adjacent. The proof is as follows. Since $u \in \mathcal{B}$, it follows that there exists a $w \in \mathcal{R}$ such that in Algorithm [2, $u \in X_{1}(w) \cup X_{2}(w)$. From Observation 24 , $u$ is adjacent to at most one vertex in $\mathcal{A}_{1}$. If it does have a neighbor in $\mathcal{A}_{1}$, it must belong to $X_{1}(w) \cup X_{2}(w)$ which is a subset of $\mathcal{B}$. Since $\mathcal{A}=\mathcal{A}_{1} \backslash \mathcal{B}, u$ is not adjacent to any vertex in $\mathcal{A}$.

Observation 4. We have some observations regarding $X_{1}(\cdot)$ and $X_{2}(\cdot)$ which are defined in Algorithm 2 , Let $v \in \mathcal{R}$.

1. $\left|X_{1}(v)\right|=2$.

2. Since $X_{1}(v) \cup X_{2}(v) \subseteq \mathcal{A}_{1}$, from Observation 24 it follows that every vertex in $X_{1}(v) \cup X_{2}(v)$ has at most one neighbor in $\mathcal{A}_{1}$ and this neighbor is in $X_{1}(v) \cup X_{2}(v)$.

3. If the two vertices in $X_{1}(v)$ are adjacent, then, $X_{2}(v)$ is empty.

4. If $X_{2}(v)$ is not empty, then, again from Observation 24, every vertex in $X_{2}(v)$ is adjacent to exactly one vertex in $X_{1}(v)$ and $\left|X_{2}(v)\right| \leq 2$.

\subsubsection{Partitioning $\mathcal{S}$ :}

We partition $\mathcal{S}$ into $\mathcal{C}$, the set of vertices which induce special cycles and $\mathcal{P}$, the set of vertices which induce special paths. $\mathcal{P}$ is further partitioned into $\mathcal{P}_{e}$, the set of end points and $\mathcal{P}_{i}$, the set of interior points of all the paths. 
Definition 3. Second end points of a path of length at least 2 are the interior vertices of the path which are adjacent to at least one of its end points.

The set of second end points of the paths in $\mathcal{P}$ is denoted by $\mathcal{P}_{2 e}$ and $\mathcal{P}_{2 i}=\mathcal{P}_{i} \backslash \mathcal{P}_{2 e}$.

Lemma 3. Every vertex in $\mathcal{N}_{1}$ is adjacent to at least one vertex in $\mathcal{C} \cup \mathcal{P}_{i}$.

Proof. Let $v \in \mathcal{N}_{1}$. Since $\mathcal{N}_{1}=N(\mathcal{S}, G), v$ is adjacent to at least one vertex in $\mathcal{S}$. If $v$ is not adjacent to any vertex in $\mathcal{C} \cup \mathcal{P}_{i}$, it implies that all its neighbors in $\mathcal{S}$ belong to $\mathcal{P}_{e}$. Let $P$ be the first path in $\mathcal{P}$ to be extracted in Algorithm 1 with $v$ as its neighbor. Since $v$ is not adjacent to any vertex in $\mathcal{P}_{i}$, it follows that it is adjacent to at least one end point of $P$ and none of its interior vertices. Since $P$ is the first component of $\mathcal{S}$ to be extracted with $v$ as the neighbor, it means that $v$ is still present in $V^{\prime}$ when $P$ is chosen as the special path. If $v$ is adjacent to both the end points of $P$, then $P \cup\{v\}$ induces a cycle in $G\left[V^{\prime}\right]$ and if $v$ is adjacent to only one end point, then $P \cup\{v\}$ induces a path in $G\left[V^{\prime}\right]$ at that stage. In either case we have a contradiction to the fact that $P$ is a special path of $G\left[V^{\prime}\right]$.

\subsection{The graph induced by $\mathcal{S} \cup \mathcal{R} \cup \mathcal{B}$}

Lemma 4. For each $u \in \mathcal{R}$, let $\Gamma(u)=\{u\} \cup X_{1}(u) \cup X_{2}(u)$, where $X_{1}(\cdot)$ and $X_{2}(\cdot)$ are as defined in Algorithm 圆. Then,

$$
\begin{aligned}
& \text { 1. } \mathcal{R} \cup \mathcal{B}=\biguplus_{u \in \mathcal{R}} \Gamma(u), \\
& \text { 2. } \Gamma(u) \text { is a component in the graph induced by } \mathcal{R} \cup \mathcal{B} \text {, and } \\
& \text { 3. } \Gamma(u) \text { is isomorphic to one of the graphs illustrated in Figure } 1 \text {. }
\end{aligned}
$$

Proof. From Algorithm 2, it is clear that $\mathcal{R} \cup \mathcal{B}=\bigcup_{u \in \mathcal{R}} \Gamma(u)$. Therefore, to prove the first statement we need to only show that for two distinct vertices $u, v \in \mathcal{R}, \Gamma(u) \cap \Gamma(v)=\varnothing$. Let us assume that $u$ was added to $\mathcal{R}$ before $v$ in the algorithm. Since any $x \in X_{1}(u) \cup X_{2}(u)$ is present in $\mathcal{B}$ when $v$ is being added to $\mathcal{R}$, it implies that $x \notin X_{1}(v) \cup X_{2}(v)$. Hence proved.

Note that $\Gamma(u)$ is connected. We will show that no vertex in $\Gamma(u)$ is adjacent to any vertex in $\Gamma(v)$, for any $v$ which was added to $\mathcal{R}$ after $u$ in Algorithm 2, Clearly, this will imply that $\Gamma(u)$ is a component in the graph induced by $\mathcal{R} \cup \mathcal{B}$. First, let us consider $u$. Since $u$ is adjacent to one vertex in $\mathcal{S}$, it has only two neighbors in $\mathcal{A}_{1}$ and these two neighbors are in $X_{1}(u)$. This implies that it is not adjacent to any vertex in $\Gamma(v)$ since $\Gamma(u) \cap \Gamma(v)=\varnothing$.

Consider any vertex $x \in X_{1}(u) \cup X_{2}(u)$. From Observation 42 and the fact that $\Gamma(u) \cap \Gamma(v)=\varnothing$, we can infer that $x$ is not adjacent to any vertex in $X_{1}(v) \cup X_{2}(v)$. Now, suppose $x$ is adjacent to $v$. Since $x \in \mathcal{B}$ when $v$ is being added to $\mathcal{R}$, we infer that $v$ is adjacent to at most one vertex in $\mathcal{A}_{1} \backslash \mathcal{B}$ at that stage. This implies that $v$ does not satisfy the condition in Line 3 in the algorithm, a contradiction since $v$ belongs to $\mathcal{R}$. Hence proved.

From Observation 4, it is easy to infer that each component $\Gamma(u)$ is isomorphic to one of the five graphs shown in Figure 1. Let $X_{1}(u)=\left\{x_{1}, x_{1}^{\prime}\right\}$. If $x_{1}$ or $x_{1}^{\prime}$ has a neighbor in $X_{2}(u)$, then, it will be denoted by $x_{2}$ or $x_{2}^{\prime}$ respectively. We have the following five graphs: In each graph, $u$ is adjacent to $x_{1}$ and $x_{1}^{\prime}$.

(a) $x_{1}$ is adjacent to $x_{1}^{\prime}$, and therefore, $X_{2}(u)=\varnothing$.

(b) $x_{1}$ is not adjacent to $x_{1}^{\prime}$ and $X_{2}(u)=\varnothing$.

(c) $x_{1}$ is not adjacent to $x_{1}^{\prime}$ and $X_{2}(u)=\left\{x_{2}\right\}$.

(d) $x_{1}$ is not adjacent to $x_{1}^{\prime}$ and $X_{2}(u)=\left\{x_{2}^{\prime}\right\}$.

(e) $x_{1}$ is not adjacent to $x_{1}^{\prime}$ and $X_{2}(u)=\left\{x_{2}, x_{2}^{\prime}\right\}$.

From Lemma 4 and Observations 33 and 314 , it follows that every vertex in $\mathcal{B}$ is adjacent to either one or two vertices in $\mathcal{R} \cup \mathcal{B}$ and no vertex in $\mathcal{S} \cup \mathcal{A}$. This implies that every vertex in $\mathcal{B}$ is adjacent to either one or two vertices in $\mathcal{N}$. Based on this, we partition $\mathcal{B}$ into two parts:

Definition 4. $\mathcal{B}_{1}$ and $\mathcal{B}_{2}: \mathcal{B}_{1}$ is the set of vertices of $\mathcal{B}$ which have one neighbor in $\mathcal{N}$ and $\mathcal{B}_{2}$ is the set of vertices of $\mathcal{B}$ which have two neighbors in $\mathcal{N}$. 


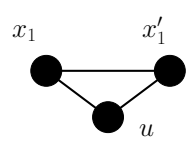

(a)

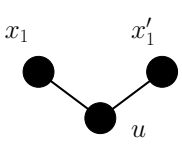

(b)

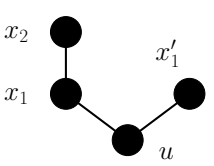

(c)

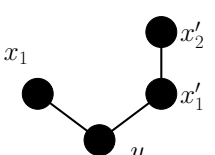

(d)

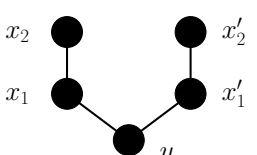

(e)

Fig. 1. In the graph induced by $\mathcal{R} \cup \mathcal{B}$, each component $\Gamma(u), u \in \mathcal{R}$ is isomorphic to one of the graphs illustrated in the figure. Here, $u$ has exactly two neighbors in $\mathcal{B}, x_{1}$ and $x_{1}^{\prime}$. These neighbors if not adjacent can each have at most one neighbor in $\mathcal{B}$ which are denoted by $x_{2}$ and $x_{2}^{\prime}$ respectively.

Recall that from Observation 312, each vertex of $\mathcal{R}$ has a unique neighbor in $\mathcal{S}$. In fact, we can infer more:

Lemma 5. Let $u \in \mathcal{R}$. The unique vertex of $\mathcal{S}$ to which $u$ is adjacent to belongs to $\mathcal{P}_{2 i}$.

Proof. Let $x$ be the unique neighbor of $u$ in $\mathcal{S}$. Let $a$ and $b$ be the remaining neighbors of $u$. From Observation 32, $a, b \in \mathcal{B}$. We need to show that $x \in \mathcal{P}_{2 i}$. We will prove by contradiction. Let $T$ be the special cycle or path in $\mathcal{S}$ which contains $x$. Since $T$ is the only component in $\mathcal{S}$ with $v$ as a neighbor, it implies that in Algorithm 1 $v$ is in $V^{\prime}$ when $T$ is being chosen as the special path or cycle. Since $a, b \in \mathcal{B}$, it implies that they belong to $\mathcal{A}_{1}$ and therefore, they too are present in $V^{\prime}$ when $T$ is being chosen. Moreover, $a$ and $b$ are not adjacent to any vertex in $T$ (Observation 313). Now, we have the following cases to consider:

$x \in \mathcal{C}$ : This implies that $T$ is a special cycle. Let $x^{\prime} \in T$ be a vertex adjacent to $x$. Clearly, $\left(T \backslash\left\{x^{\prime}\right\}\right) \cup\{v, a\}$ induces a path of length $|T|+1$ in $G\left[V^{\prime}\right]$ contradicting the fact that $T$ is a special cycle.

$x \in \mathcal{P}_{e}$ : This implies that $T$ is a special path. Since $v$ is not adjacent to any other vertex in the path, $T \cup\{v\}$ induces a path of length $|T|+1$ in $G\left[V^{\prime}\right]$, contradicting its maximality and thus $T$ cannot be a special path.

$x \in \mathcal{P}_{2 e}$ : Again, this implies that $T$ is a special path. Let $x_{e}$ be an end point of $T$ to which $x$ is adjacent to. Clearly, $\left(T \backslash\left\{x_{e}\right\}\right) \cup\{v, a\}$ induces a path of length $|T|+1$ in $G\left[V^{\prime}\right]$, contradicting the fact that $T$ is a special path.

Therefore, $x \in \mathcal{P}_{2 i}$.

Observation 5. We have the following observations due to Lemma 5.

1. Each vertex in $\mathcal{C} \cup \mathcal{P}_{2 e}$ is adjacent to exactly one vertex in $\mathcal{N}$. The proof is as follows: Note that each vertex in $\mathcal{C} \cup \mathcal{P}_{2 e}$ either belongs to a special cycle or is an interior vertex of a special path in $G[\mathcal{C} \cup \mathcal{P}]$. Therefore, it has only one neighbor in $V \backslash(\mathcal{C} \cup \mathcal{P})$ and by Observation 202, it must belong to $\mathcal{N}_{1}$. By Lemma 5, it does not belong to $\mathcal{R}$. Hence, it belongs to $\mathcal{N}$.

2. Every vertex in $\mathcal{P}_{e}$ is adjacent to exactly two vertices in $\mathcal{N}$.

3. Every vertex in $\mathcal{P}_{2 i}$ is adjacent to exactly one vertex in $\mathcal{R} \cup \mathcal{N}=\mathcal{N}_{1}$.

\subsection{The graph induced by $\mathcal{B}_{2} \cup \mathcal{P}_{e} \cup \mathcal{N}$}

Lemma 6. $\mathcal{B}_{2} \cup \mathcal{P}_{e}$ is an independent set in $G$.

Proof. Let $x, y \in \mathcal{P}_{e}$. If $x$ and $y$ are the end points of two different paths in $\mathcal{P}$, clearly, they are not adjacent. Since each path is special, it has at least 3 vertices which implies that if $x$ and $y$ are end points of the same path, then they are not adjacent. Hence, $\mathcal{P}_{e}$ induces an independent set in $G$. Let $x, y \in \mathcal{B}_{2}$. By definition, they have two neighbors each in $\mathcal{N}$. Therefore, if $x$ and $y$ are adjacent, they induce a component in $\mathcal{R} \cup \mathcal{B}$, which contradicts Statement 3 of Lemma 4 . Noting that $\mathcal{P}_{e} \subseteq \mathcal{S}$ and $\mathcal{B}_{2} \subseteq \mathcal{B}$, from Observation 33, it follows that no vertex in $\mathcal{B}_{2}$ is adjacent to any vertex in $\mathcal{P}_{e}$. Hence proved. 
Observation 6. Consider a vertex $v \in \mathcal{B}_{2} \cup \mathcal{P}_{e}$. By the definition of $\mathcal{B}_{2}$ and Observation 52, it follows that $v$ is adjacent to exactly two vertices in $\mathcal{N}$. By Lemma 6 , $v$ is not adjacent to any vertex in $\mathcal{B}_{2} \cup \mathcal{P}_{e}$. Therefore, in the graph induced by $\mathcal{B}_{2} \cup \mathcal{P}_{e} \cup \mathcal{N}$, its degree is 2. Lemma 3 implies that every vertex in $\mathcal{N}$ is adjacent to at most two vertices in $\mathcal{B}_{2} \cup \mathcal{P}_{e} \cup \mathcal{N}$. From these two observations, we can infer the following about the graph induced by $\mathcal{B}_{2} \cup \mathcal{P}_{e} \cup \mathcal{N}$.

1. Its maximum degree is 2 and thus is a collection of paths and cycles.

2. All the end points of paths (which also includes isolated vertices) belong to $\mathcal{N}$.

3. A vertex in $\mathcal{N}$ is adjacent to a vertex in $\mathcal{A}$ only if it is an end point of a path. The proof is as follows: Let $v \in \mathcal{N}$. It has at least one neighbor in $S$ (Observation 22). If it has a neighbor in $\mathcal{A}$, then it can have at most one neighbor in $\mathcal{B}_{2} \cup \mathcal{P}_{e} \cup \mathcal{N}$. Hence, proved.

Definition 5. $\mathcal{N}_{e}$ and $\mathcal{N}_{\text {int }}: \mathcal{N}$ is partitioned into $\mathcal{N}_{e}$, the set of end points of paths (which includes isolated vertices) and $\mathcal{N}_{\text {int }}$, the set of interior points of cycles and paths in $G\left[\mathcal{B}_{2} \cup \mathcal{P}_{e} \cup \mathcal{N}\right]$.

In view of Observation 613, a vertex in $\mathcal{N}$ is adjacent to a vertex in $\mathcal{A}$ only if it belongs to $\mathcal{N}_{e}$.

Definition 6. Type 1 and Type 2 cycles: Recall that by Observation 6.1, $\mathcal{B}_{2} \cup \mathcal{P}_{e} \cup \mathcal{N}$ induces a collection of cycles and paths. We classify the cycles in the following manner:

Type 1: Cycles whose vertices alternate between $\mathcal{N}$ and $\mathcal{B}_{2} \cup \mathcal{P}_{e}$.

Type 2: Cycles which are not Type 1. From Lemma [6, it is easy to infer that such a cycle has at least one pair of adjacent vertices which belong to $\mathcal{N}$.

Lemma 7. If a vertex $v \in \mathcal{N}$ is adjacent to 2 vertices in $\mathcal{B}_{2} \cup \mathcal{P}_{e}$, then its remaining neighbor belongs to $\mathcal{P}_{2 i}$. In other words, $v$ has no neighbor in $\mathcal{C} \cup \mathcal{P}_{2 e}$.

Proof. Let $v \in \mathcal{N}$ be such that it is adjacent to two vertices in $\mathcal{B}_{2} \cup \mathcal{P}_{e}$. Let $x_{1}$ and $x_{2}$ be these neighbors. From Lemma 3, it follows that $v$ is adjacent to one vertex in $\mathcal{C} \cup \mathcal{P}_{i}$. Let this vertex be $y$. We need to show that $y \in \mathcal{P}_{2 i}$.

Suppose $T$ is the first special path or cycle chosen in Algorithm 1 with $v$ as a neighbor. We will first show that $v, x_{1}$ and $x_{2}$ are present in $V^{\prime}$ when $T$ is being chosen. Since $T$ is the first component of $\mathcal{S}$ with $v$ as its neighbor, it implies that $v \in V^{\prime}$ at that time. If any of $x_{1}$ and $x_{2}$ belongs to $\mathcal{B}_{2}$, then, it is in $V^{\prime}$ because $\mathcal{B}_{2} \subseteq \mathcal{A}_{1} \subseteq V^{\prime}$ at any time in the algorithm. If any of $x_{1}$ and $x_{2}$, say $x$ is in $\mathcal{P}_{e}$, then again, since $T$ is the first component of $\mathcal{S}$ with $v$ as the neighbor, it follows that $x$ is an end point of either $T$ or a special path chosen after $T$. In either case, $x$ belongs to $V^{\prime}$ when $T$ is being chosen. The following observation is crucial for the proof:

Consider any $t \in\left\{y, x_{1}, x_{2}\right\}$. If $t \notin T$, it implies that it is not adjacent to any vertex in $T$, since otherwise it would be present in $\mathcal{N}_{1}$ and this is not possible since by assumption $y \in \mathcal{S}$ and $x_{1}, x_{2} \in \mathcal{B}_{2} \cup \mathcal{P}_{e}$.

We will now show that if $y \in \mathcal{C} \cup \mathcal{P}_{2 e}$, it contradicts the assumption that $T$ is a special cycle or path.

Let us suppose that $T$ is a special cycle. Since $x_{1}, x_{2} \notin \mathcal{C}$, it follows that $x_{1}, x_{2} \notin T$ and therefore, $y \in T$. Let $y^{\prime} \in T$ be adjacent to $y$. Since $x_{1} \notin T$, it is not adjacent to any vertex of $T$ and therefore, $\left(T \backslash\left\{y^{\prime}\right\}\right) \cup\left\{v, x_{1}\right\}$ induces a path with $|T|+1$ vertices in $G\left[V^{\prime}\right]$ contradicting the fact that $T$ is a special cycle. Therefore, from now on we will assume that $T$ is a special path.

If $y \notin T$, it implies that at least one of $\left\{x_{1}, x_{2}\right\}$ belongs to $T$. If only one of them, say $x_{1} \in T$, then, since $x_{1}$ has to be an end point of $T, T \cup\{v\}$ induces a longer path in $G\left[V^{\prime}\right]$ and if both $x_{1}, x_{2} \in T$, then, $T \cup\{v\}$ induces a cycle. In either case, we have a contradiction to the fact that $T$ is a special path.

Suppose $y \in T$. Since we have assumed that $y \in \mathcal{C} \cup \mathcal{P}_{2 e}$, this means $y$ is a second end point of $T$. Let $p_{e}^{\prime}$ be an end point of $T$ adjacent to $y$ and let $p_{e}$ be the other end point. If $p_{e} \in\left\{x_{1}, x_{2}\right\}$, then, $\left(T \backslash\left\{p_{e}^{\prime}\right\}\right) \cup\{v\}$ induces a cycle contradicting the fact that $T$ is a special path. If $p_{e} \notin\left\{x_{1}, x_{2}\right\}$, it implies that at most one vertex from $\left\{x_{1}, x_{2}\right\}$ can be an end point of $T$. Without loss of generality, we assume that $x_{1}$ is not an end point of $T$. This implies that $x_{1} \notin T$ and therefore, is not adjacent to any vertex in $T$. Hence, $\left(T \backslash\left\{p_{e}^{\prime}\right\}\right) \cup\left\{v, x_{1}\right\}$ induces a path with $|T|+1$ vertices, again contradicting the fact that $T$ is a special path. Therefore, $y \in \mathcal{P}_{2 i}$.

Observation 7. We can assume that $\left|\mathcal{B}_{2} \cup \mathcal{P}_{e} \cup \mathcal{N}\right| \leq n-2$. This is because, since $G$ is cubic, it has a cycle and therefore, we can always extract a special cycle with at least 3 vertices or a special path with at least 4 vertices from $V$. Thus, we can ensure that $\left|\mathcal{S} \backslash \mathcal{P}_{e}\right| \geq 2$. This implies that, $\left|\mathcal{B}_{2} \cup \mathcal{P}_{e} \cup \mathcal{N}\right| \leq n-2$. 


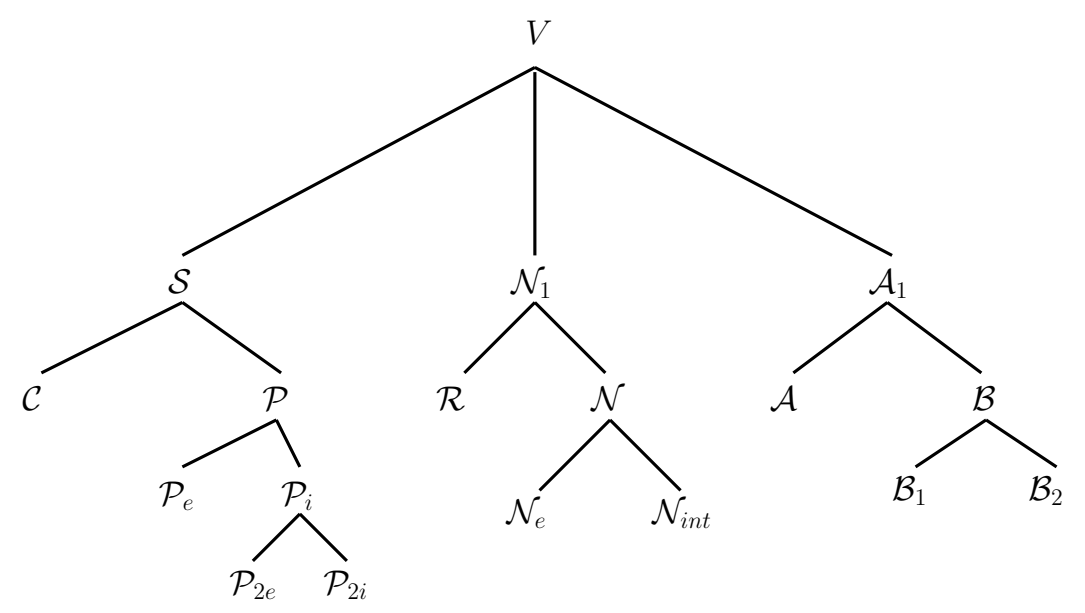

Fig. 2. The partition of the vertex set $V$ of the cubic graph. The first and second level partitions are due to Algorithms 1 and 2 respectively. The partitioning of $\mathcal{P}$ is covered in the beginning of Section 2.2.1. The partitioning of $\mathcal{N}$ and $\mathcal{B}$ are due to Definitions 5 and 4 respectively.

\begin{tabular}{|c|c|c|c|}
\hline 1 & $\overline{\mathcal{A}}$ & $\mathcal{C} \cup \mathcal{P}$ & using Observation 233 and the fact that $\mathcal{A} \subseteq \mathcal{A}_{1}$ \\
\hline 2 & $\overline{\mathcal{A} \cup \mathcal{N}}$ & $\mathcal{R}$ & Observation 32 (b) \\
\hline 3 & $\overline{\mathcal{A}}$ & $\overline{\mathcal{B}}$ & Observation 34 \\
\hline 4 & $\mathcal{C}$ & $\mathcal{P}$ & follows directly from the definitions of $\mathcal{C}$ and $\mathcal{P}$. \\
\hline & $\mathcal{C} \cup \mathcal{P}_{e} \cup \mathcal{P}_{2 e}$ & $\overline{\mathcal{R}}$ & Lemma 5 \\
\hline 6 & $\mathcal{C} \cup \mathcal{P}$ & $\mathcal{B}$ & Observation 33 \\
\hline 7 & $\overline{\mathcal{P}_{e}}$ & $\overline{\mathcal{P}_{e}}$ & Lemma 6 \\
\hline 8 & $\overline{\mathcal{P}_{e}}$ & $\mathcal{P}_{2 i}$ & follows directly from the definitions of $\mathcal{P}_{e}$ and $\mathcal{P}_{2 i}$ \\
\hline 9 & $\overline{\mathcal{N}_{\text {int }}}$ & $\mathcal{A}$ & Observation $6[3$ and Definition 5 \\
\hline 10 & $\overline{\mathcal{R}}$ & $\mathcal{R}$ & Observation $3 / 2$ (a) \\
\hline
\end{tabular}

Table 1. Non-adjacency table: In every row, there is no edge between the set in the 1st column and the set in the 2nd column in $G$.

\subsection{A summary}

In this section, we partitioned the vertex set $V$ as follows: $V=\mathcal{S} \uplus \mathcal{N}_{1} \uplus \mathcal{A}_{1}$, where $\mathcal{S}=\mathcal{C} \uplus \mathcal{P}, \mathcal{N}_{1}=\mathcal{R} \uplus \mathcal{N}$ and $\mathcal{A}_{1}=\mathcal{B} \uplus \mathcal{A}$. Further, $\mathcal{P}=\mathcal{P}_{e} \uplus \mathcal{P}_{2 e} \uplus \mathcal{P}_{2 i}$ and $\mathcal{B}=\mathcal{B}_{1} \uplus \mathcal{B}_{2}$. Therefore, $V=\mathcal{C} \uplus \mathcal{P}_{e} \uplus \mathcal{P}_{2 e} \uplus \mathcal{P}_{2 i} \uplus \mathcal{N} \uplus \mathcal{R} \uplus \mathcal{B}_{1} \uplus \mathcal{B}_{2} \uplus \mathcal{A}$. This partitioning of $V$ is illustrated in Figure 2. Some of the observations and lemmas which we developed will be frequently referred to in the sections to come. For the convenience of the reader, we have tabulated them as follows. In Table 1, we have listed the pairs of sets $X, Y \subseteq V$ which satisfy the property that in $G$ there is no edge between $X$ and $Y$. The relevant observation or lemma is listed in the third column. In Table 2, we list pairs of sets $X, Y \subseteq V$ such that in $G$, every vertex of $X$ has at most one neighbor in $Y$. The corresponding observation or lemma can be found in the third column. In the fourth column, we give information on whether the vertex in $X$ has at most one neighbor or exactly one neighbor in $Y$.

\section{Construction of a 3-box representation of $G$}

In order to give a 3-box representation of $G$, we define three interval graphs $I_{1}, I_{2}$ and $I_{3}$ and verify that $E(G)=E\left(I_{1}\right) \cap E\left(I_{2}\right) \cap E\left(I_{3}\right)$. Let $n=|V|$, the number of vertices in $G$. For any $v \in V$, let $f\left(v, I_{j}\right)$, $j=1,2,3$ denote the closed interval assigned to $v$ in the interval representation of $I_{j}$. Further, let $l\left(v, I_{j}\right)$ and $r\left(v, I_{j}\right)$ denote the left and right end points of $f\left(v, I_{j}\right)$ respectively. In each interval graph, the interval assigned to a vertex is based on the set it belongs to in the partition of $V$ (illustrated in Figure 21). We will 


\begin{tabular}{|l|c|c|l|l|}
\hline 1 & $\mathcal{N}_{e}$ & $\mathcal{A}$ & Observation $[6] 3$ and Definition 5 & at most one neighbor \\
\hline 2 & $\mathcal{B}_{1}$ & $\mathcal{N}$ & Definition 4 & exactly one neighbor \\
\hline 3 & $\mathcal{C} \cup \mathcal{P}_{2 e}$ & $\mathcal{N}$ & Observation $5[1]$ & exactly one neighbor \\
\hline 4 & $\mathcal{P}_{2 i}$ & $\mathcal{R} \cup \mathcal{N}$ & Observation $5[3$ & exactly one neighbor \\
\hline 5 & $\mathcal{R}$ & $\mathcal{P}_{2 i}$ & Lemma 5 & exactly one neighbor \\
\hline
\end{tabular}

Table 2. Unique neighbor table: In every row, each vertex belonging to the set in the 1 st column has either (a) at most one neighbor OR (b) exactly one neighbor in the set given in the 2nd column.

also show that for every pair of adjacent vertices $x$ and $y$, in at least one interval graph $I_{j}, j \in\{1,2,3\}$, either $l\left(x, I_{j}\right)=r\left(y, I_{j}\right)$ or $l\left(y, I_{j}\right)=r\left(x, I_{j}\right)$. This is sufficient to prove that their corresponding boxes intersect only at their boundaries.

\subsection{Construction of $I_{1}$}

\subsubsection{Vertices of $\mathcal{A}$}

We recall from Observation 24 that since $\mathcal{A} \subseteq \mathcal{A}_{1}$, it induces a collection of isolated vertices and edges in $G$.

Definition 7. Let $\Pi_{A}$ be an ordering of $\mathcal{A}$ which satisfies the condition that the two end points of every (isolated) edge are consecutively ordered.

The intervals assigned to the vertices of $\mathcal{A}$ are as follows:

An isolated vertex $u$ is given a point interval as follows:

$$
f\left(u, I_{1}\right)=\left[2 n+\Pi_{A}(u), 2 n+\Pi_{A}(u)\right] .
$$

End points of an isolated edge $(u, v)$ : Without loss of generality, let $\Pi_{A}(v)=\Pi_{A}(u)+1$. We assign the intervals to $u$ and $v$ as follows:

$$
\begin{aligned}
& f\left(u, I_{1}\right)=\left[2 n+\Pi_{A}(u), 2 n+\Pi_{A}(u)+0.5\right], \\
& f\left(v, I_{1}\right)=\left[2 n+\Pi_{A}(v)-0.5,2 n+\Pi_{A}(v)\right] .
\end{aligned}
$$

Observation 8. Let $x, y \in \mathcal{A}$ such that $\Pi_{A}(x)<\Pi_{A}(y)$. If they are adjacent in $G$, then, $r\left(x, I_{1}\right)=l\left(y, I_{1}\right)$. This follows from (2) and (3).

Lemma 8. The graph induced by $\mathcal{A}$ in $I_{1}$ and $G$ are identical, that is, $I_{1}[\mathcal{A}]=G[\mathcal{A}]$.

Proof. From the interval assignments (1), (2) and (3), we observe the following: for any $x \in \mathcal{A}$, (a) the point $2 n+\Pi_{A}(x)$ is an end point of $f\left(x, I_{1}\right)$ and (b) either $f\left(x, I_{1}\right)$ is a point interval or its length is 0.5 .

Suppose $x, y \in \mathcal{A}$ such that $\Pi_{A}(x)<\Pi_{A}(y)$. From the above observations it follows that $x$ and $y$ are adjacent in $I_{1}$ if and only if $\Pi_{A}(y)=\Pi_{A}(x)+1$ and $f\left(x, I_{1}\right)=\left[2 n+\Pi_{A}(x), 2 n+\Pi_{A}(x)+0.5\right]$ and $f\left(y, I_{1}\right)=\left[2 n+\Pi_{A}(y)-0.5,2 n+\Pi_{A}(y)\right]$. This implies that $x$ was assigned an interval by (2) and $y$, by (3) . This happens only if $x$ and $y$ are the end points of an isolated edge in $G[\mathcal{A}]$. Hence proved.

\subsubsection{Vertices of $\mathcal{B}_{2} \cup \mathcal{P}_{e} \cup \mathcal{N}$}

We define an ordering on this set.

Definition 8. Let $\Pi_{1}$ be an ordering of $\mathcal{B}_{2} \cup \mathcal{P}_{e} \cup \mathcal{N}$ which satisfies the following properties. Let $S$ be a component induced by $\mathcal{B}_{2} \cup \mathcal{P}_{e} \cup \mathcal{N}$. We recall from Section 2.4 that $S$ is either a path or a cycle. 
1. Let $S$ be a path with at least two vertices. Then, for one of the natural orderings of the vertices of $S$, say $p_{1} p_{2} \ldots p_{t}$, we have $\Pi_{1}\left(p_{i}\right)=\Pi_{1}\left(p_{i-1}\right)+1,2 \leq i \leq t$.

2. Suppose $S$ is a cycle. We recall that $S$ is either a Type 1 or a Type 2 cycle (Definition 6 ). Consider one of the natural orderings of the vertices of $S$, say $c_{1} c_{2} \ldots c_{t} c_{1}$ such that if $S$ is a Type 1 cycle, then $c_{1} \in \mathcal{N}$ and if it is a Type 2 cycle, then $c_{1}, c_{t} \in \mathcal{N}$. Then, we have, $\Pi_{1}\left(c_{i}\right)=\Pi_{1}\left(c_{i-1}\right)+1,2 \leq i \leq t$.

3. Let $\mathcal{B}_{2} \cup \mathcal{P}_{e} \cup \mathcal{N}=\Lambda_{\text {Type } 1} \uplus \Lambda_{\text {Type } 2} \uplus \Lambda_{\text {Paths }}$ where, $\Lambda_{\text {Type } 1}, \Lambda_{\text {Type } 2}$ and $\Lambda_{\text {Paths }}$ are the sets of vertices belonging to Type 1 cycles, Type 2 cycles and paths respectively. Then, we have $\Pi_{1}\left(\Lambda_{\text {Type } 1)<}\right.$ $\Pi_{1}\left(\Lambda_{\text {Type } 2}\right)<\Pi_{1}\left(\Lambda_{\text {Paths }}\right)$.

It is easy to verify that such an ordering exists. We also infer that if $S_{1}$ and $S_{2}$ are two different components of $G\left[\mathcal{B}_{2} \cup \mathcal{P}_{e} \cup \mathcal{N}\right]$, then, either $\Pi_{1}\left(S_{1}\right)<\Pi_{1}\left(S_{2}\right)$ or $\Pi_{1}\left(S_{2}\right)<\Pi_{1}\left(S_{1}\right)$.

Observation 9. $\forall z \in \mathcal{B}_{2} \cup \mathcal{P}_{e} \cup \mathcal{N}, \Pi_{1}(z)<n-1$. This follows from the fact that $\left|\mathcal{B}_{2} \cup \mathcal{P}_{e} \cup \mathcal{N}\right|<n-1$ (Observation 7).

The interval assignments for the vertices of $\mathcal{B}_{2} \cup \mathcal{P}_{e} \cup \mathcal{N}$ are as follows:

For a vertex in a Type 1 cycle: Let $S=c_{1} c_{2} \ldots c_{t} c_{1}$ be a Type 1 cycle such that $\Pi_{1}\left(c_{i+1}\right)=\Pi_{1}\left(c_{i}\right)+1$, $1 \leq i<t$.

$$
\begin{aligned}
& f\left(c_{1}, I_{1}\right)=\left[\Pi_{1}\left(c_{1}\right), \Pi_{1}\left(c_{t}\right)\right] ; \\
& f\left(c_{i}, I_{1}\right)=\left[\Pi_{1}\left(c_{i}\right), \Pi_{1}\left(c_{i}\right)+1\right], 1<i<t ; \\
& f\left(c_{t}, I_{1}\right)=\left[\Pi_{1}\left(c_{t}\right), \Pi_{1}\left(c_{t}\right)+0.5\right] .
\end{aligned}
$$

Observation 10. Suppose $S=c_{1} c_{2} \ldots c_{t} c_{1}$ is a Type 1 cycle such that $\Pi_{1}\left(c_{i+1}\right)=\Pi_{1}\left(c_{i}\right)+1,1 \leq i<t$.

1. For $1<i<t, r\left(c_{i}, I_{1}\right)=l\left(c_{i+1}, I_{1}\right)$ and therefore, $I_{1}\left[S \backslash c_{1}\right]=G\left[S \backslash c_{1}\right]$. This is because, from (5) and (6), $r\left(c_{i}, I_{1}\right)=\Pi_{1}\left(c_{i}\right)+1=\Pi_{1}\left(c_{i+1}\right)=l\left(c_{i+1}, I_{1}\right)$.

2. $I_{1}[S]$ is a supergraph of $G[S]$. The proof is as follows: $c_{1}$ is adjacent to all the other vertices of $S$. This follows from (4): $l\left(c_{i}, I_{1}\right)=\Pi_{1}\left(c_{i}\right) \in f\left(c_{1}, I_{1}\right)$. From Point $1, c_{i}$ is adjacent to $c_{i+1}, 2 \leq i<t$. Hence, proved.

3. Let $x \in S_{x}$ and $y \in S_{y}$, where $S_{x}$ and $S_{y}$ induce different Type 1 cycles. Then, $(x, y) \notin E\left(I_{1}\right)$. The proof is as follows: Without loss of generality, let $\Pi_{1}\left(S_{x}\right)<\Pi_{1}\left(S_{y}\right)$. From (4-6), it follows that $r\left(x, I_{1}\right) \leq$ $\max _{a \in S_{x}} \Pi_{1}(a)+0.5<\min _{b \in S_{y}} \Pi_{1}(b) \leq l\left(y, I_{1}\right)$. Therefore, $(x, y) \notin E\left(I_{1}\right)$.

For a vertex in a Type 2 cycle: Let $S=c_{1} c_{2} \ldots c_{t} c_{1}$ be a Type 2 cycle such that $\Pi_{1}\left(c_{i+1}\right)=\Pi_{1}\left(c_{i}\right)+1$, $1 \leq i<t$. We recall from the definition of $\Pi_{1}$ that $c_{1}, c_{t} \in \mathcal{N}$. They are assigned intervals as follows:

$$
\begin{aligned}
f\left(c_{1}, I_{1}\right) & =\left[n+\Pi_{1}\left(c_{1}\right), n+\Pi_{1}\left(c_{t}\right)\right], \\
f\left(c_{t}, I_{1}\right) & =\left[n+\Pi_{1}\left(c_{t}\right), n+\Pi_{1}\left(c_{t}\right)+0.5\right] .
\end{aligned}
$$

The remaining vertices are assigned intervals as follows. For $1<i<t$,

$$
\begin{aligned}
& \text { if } c_{i} \in \mathcal{N} \text {, then, } f\left(c_{i}, I_{1}\right)=\left[n+\Pi_{1}\left(c_{i}\right), n+\Pi_{1}\left(c_{i}\right)+1\right] \text {, } \\
& \text { if } c_{i} \in \mathcal{B}_{2} \cup \mathcal{P}_{e} \text {, then, } f\left(c_{i}, I_{1}\right)=\left[n, n+\Pi_{1}\left(c_{i}\right)+1\right] .
\end{aligned}
$$

Observation 11. Let $S=c_{1} c_{2} \ldots c_{t} c_{1}$ be a Type 2 cycle such that $\Pi_{1}\left(c_{i+1}\right)=\Pi_{1}\left(c_{i}\right)+1,1 \leq i<t$.

1. $I_{1}[S]$ is a supergraph of $G[S]$. The proof is as follows: From (710), $\forall c \in S, n+\Pi_{1}(c) \in f\left(c, I_{1}\right)$. From (7), we note that $\forall c \in S, n+\Pi_{1}(c) \in f\left(c_{1}, I_{1}\right)$ and therefore, $c_{1}$ is adjacent to all the other vertices of $S$. From (9) and (10), for $1<i<t, r\left(c_{i}, I_{1}\right)=n+\Pi_{1}\left(c_{i}\right)+1=n+\Pi_{1}\left(c_{i+1}\right) \in f\left(c_{i+1}, I_{1}\right)$. Therefore, $c_{i}$ is adjacent to $c_{i+1}, 1<i<t$.

2. Let $x, y \in S$ be two adjacent vertices in $G$ such that neither $x$ nor $y$ is $c_{1}$. If $\Pi_{1}(x)>\Pi_{1}(y)$ and $x \in \mathcal{N}$, then, $l\left(x, I_{1}\right)=r\left(y, I_{1}\right)$. This follows by noting that $\Pi_{1}(x)=\Pi_{1}(y)+1$ and subsequently applying it in (8) 
For a vertex in a path: Let $S=p_{1} p_{2} \ldots p_{t}$ be a path such that $\Pi_{1}\left(c_{i+1}\right)=\Pi_{1}\left(c_{i}\right)+1,1 \leq i<t$. By Definition [5, $p_{1}, p_{t} \in \mathcal{N}_{e}$. From Table 2 (row 1), they can be adjacent to at most one vertex in $\mathcal{A}$. Taking this into consideration, the interval assignments are as follows: Let $p \in\left\{p_{1}, p_{t}\right\} \subseteq \mathcal{N}_{e}$ :

if $p$ is not adjacent to any vertex in $\mathcal{A}$, then, $f\left(p, I_{1}\right)=\left[n+\Pi_{1}(p), 2 n\right]$,

if $p$ is adjacent to a vertex $a$ in $\mathcal{A}$, then, $f\left(p, I_{1}\right)=\left[n+\Pi_{1}(p), l\left(a, I_{1}\right)\right]$.

Note that $f\left(a, I_{1}\right)$ is already defined in (1) 3 . Moreover, $l\left(a, I_{1}\right)>2 n \geq n+\Pi_{1}(p)$. Therefore, $f\left(p, I_{1}\right)$ is well-defined in (12). If $p$ is an interior point of the path, its interval assignment is as follows:

$$
\begin{aligned}
& \text { if } p \in \mathcal{N}_{\text {int }} \text {, then, } f\left(p, I_{1}\right)=\left[n+\Pi_{1}(p), n+\Pi_{1}(p)+1\right], \\
& \text { if } p \in \mathcal{B}_{2} \cup \mathcal{P}_{e} \text {, then, } f\left(p, I_{1}\right)=\left[n, n+\Pi_{1}(p)+1\right] .
\end{aligned}
$$

Observation 12. Let $S=p_{1} p_{2} \ldots p_{t}$ be a path such that $\Pi_{1}\left(c_{i+1}\right)=\Pi_{1}\left(c_{i}\right)+1,1 \leq i<t$.

1. $I_{1}[S]$ is a supergraph of $G[S]$. The proof is as follows: For all $p \in S, n+\Pi_{1}(p), n+\Pi_{1}(p)+1 \in f\left(p, I_{1}\right)$. This is easy to infer from (11 14) and the fact that $\Pi_{1}(p)<n-1$ (Observation 9). This implies that for $1 \leq i<t, p_{i}$ is adjacent to $p_{i+1}$. Hence, proved.

2. Let $x, y \in S$ be two adjacent vertices in $G$. If $\Pi_{1}(x)>\Pi_{1}(y), x \in \mathcal{N}$ and $y \in \mathcal{B}_{2} \cup \mathcal{P}_{e}$, then, $l\left(x, I_{1}\right)=$ $r\left(y, I_{1}\right)$. This follows by noting that $\Pi_{1}(x)=\Pi_{1}(y)+1$ and subsequently applying it in (11) 14. .

Observation 13. We have some observations regarding the intervals assigned to vertices of $\mathcal{B}_{2} \cup \mathcal{P}_{e} \cup \mathcal{N}$. We repeatedly make use of Observation 9.

1. If $z$ belongs to a Type 1 cycle, then, (a) $l\left(z, I_{1}\right)=\Pi_{1}(z)$ and (b) $1 \leq l\left(z, I_{1}\right)<r\left(z, I_{1}\right)<n$. The proof is as follows: Let $z$ belong to the Type 1 cycle $S_{z}=c_{1} c_{2} \ldots c_{t} c_{1}$ such that $\Pi_{1}\left(c_{i+1}\right)=\Pi_{1}\left(c_{i}\right)+1,1 \leq i<t$. From (4-6), it immediately follows that $l\left(z, I_{1}\right)=\Pi_{1}(z), l\left(z, I_{1}\right)<r\left(z, I_{1}\right)$ and $r\left(z, I_{1}\right) \leq \Pi_{1}\left(c_{t}\right)+0.5<$ $(n-1)+0.5<n$.

2. If $z \in \mathcal{N}$ belongs to a Type 2 cycle or a path then, $l\left(z, I_{1}\right)=n+\Pi_{1}(z)$ and therefore, $n<l\left(z, I_{1}\right)<2 n-1$. This follows from (79) for a Type 2 cycle and (11 13) for a path.

3. If $z \in \mathcal{B}_{2} \cup \mathcal{P}_{e}$ belongs to a Type 2 cycle or a path then, $l\left(z, I_{1}\right)=n$ and $r\left(z, I_{1}\right)=n+\Pi_{1}(z)+1<2 n$. This follows from (10) for a Type 2 cycle and (14) for a path..

4. If $x, y \in \mathcal{N}$ such that $\Pi_{1}(x)<\Pi_{1}(y)$, then, $l\left(x, I_{1}\right)+1 \leq l\left(y, I_{1}\right)$. The proof is as follows: If $x$ and $y$ belong to Type 1 cycles, then by Point 1 in this observation, $l\left(x, I_{1}\right)+1=\Pi_{1}(x)+1 \leq \Pi_{1}(y)=l\left(y, I_{1}\right)$. If $y$ belongs to a Type 1 cycle, then by Definition 8 (Point 3), $x$ also belongs to a Type 1 cycle. Therefore, it is not possible that $y$ is in a Type 1 cycle and $x$ is not. If $x$ is in a Type 1 cycle and $y$ is not, then, $l\left(x, I_{1}\right)+1=\Pi_{1}(x)+1<n+\Pi_{1}(y)=l\left(y, I_{1}\right)$. Finally, if both belong to a Type 2 cycle or a path, then, $l\left(x, I_{1}\right)+1=n+\Pi_{1}(x)+1 \leq n+\Pi_{1}(y)=l\left(y, I_{1}\right)$ (from Point 2 in this observation).

5. If $x$ belongs to a Type 1 cycle and $y$ belongs to either a Type 2 cycle or a path, then, $x$ and $y$ are not adjacent in $I_{1}$. The proof is as follows: From Point 1 in this observation, $r\left(x, I_{1}\right)<n$ and from Points 2 and [3, $l\left(y, I_{1}\right) \geq n$.

6. If $x \in \mathcal{N}$ is adjacent to $a \in \mathcal{A}$, then, $r\left(x, I_{1}\right)=l\left(a, I_{1}\right)$. The proof is as follows: By Table 1 (row 9), $x \in \mathcal{N}_{e}$ and by Table 2 (row 1), a should be the only neighbor of $x$ in $\mathcal{A}$. The interval assignment for $x$ is given in (12) where, $r\left(x, I_{1}\right)=l\left(a, I_{1}\right)$.

Lemma 9. $I_{1}\left[\mathcal{A} \cup \mathcal{B}_{2} \cup \mathcal{P}_{e} \cup \mathcal{N}\right]$ is a supergraph of $G\left[\mathcal{A} \cup \mathcal{B}_{2} \cup \mathcal{P}_{e} \cup \mathcal{N}\right]$.

Proof. Let $x, y \in \mathcal{A} \cup \mathcal{B}_{2} \cup \mathcal{P}_{e} \cup \mathcal{N}$ be two adjacent vertices in $G$. If $x, y \in \mathcal{A}$, then, by Lemma 8 they are adjacent in $I_{1}$. Let $x, y \in \mathcal{B}_{2} \cup \mathcal{P}_{e} \cup \mathcal{N}$. They have to belong to the same component in $G\left[\mathcal{B}_{2} \cup \mathcal{P}_{e} \cup \mathcal{N}\right]$, which is either a Type 1 cycle, Type 2 cycle or a path. By Observations 102, 111 and 121, $x$ and $y$ are adjacent in $I_{1}$.

Now it remains to be shown that if $x \in \mathcal{A}$ and $y \in \mathcal{B}_{2} \cup \mathcal{P}_{e} \cup \mathcal{N}$, then they are adjacent in $I_{1}$. Noting that $\mathcal{B}_{2} \subseteq \mathcal{B}$ and $\mathcal{P}_{e} \subseteq \mathcal{P}, y \notin \mathcal{B}_{2} \cup \mathcal{P}_{e}$ (see Table 1 , rows 1 and 3). Therefore, $y \in \mathcal{N}$. By Observation 136 $r\left(y, I_{1}\right)=l\left(x, I_{1}\right)$. Therefore, $x$ is adjacent to $y$ in $I_{1}$. Hence proved. 


\subsubsection{Vertices of $\mathcal{B}_{1} \cup \mathcal{P}_{2 e}$}

Let $v \in \mathcal{B}_{1} \cup \mathcal{P}_{2 e}$. From Table 2 (rows 2 and 3), we note that $v$ has a unique neighbor in $\mathcal{N}$, say $v^{\prime} . f\left(v^{\prime}, I_{1}\right)$ is already defined in Section 3.1 .2 .

$$
\begin{aligned}
& \text { if } v \in \mathcal{B}_{1} \text {, then, } f\left(v, I_{1}\right)=\left[0, l\left(v^{\prime}, I_{1}\right)\right], \\
& \text { if } v \in \mathcal{P}_{2 e} \text {, then, } f\left(v, I_{1}\right)=\left[-1, l\left(v^{\prime}, I_{1}\right)\right] .
\end{aligned}
$$

Lemma 10. For any $x \in \mathcal{B}_{1} \cup \mathcal{P}_{2 e}, r\left(x, I_{1}\right)>n$ and therefore, $[0, n] \subset f\left(x, I_{1}\right)$.

Proof. From Table 2 (rows 2 and 3), $x$ has a unique neighbor in $\mathcal{N}$, say $x^{\prime}$. From (15) and (16), $r\left(x, I_{1}\right)=$ $l\left(x^{\prime}, I_{1}\right)$. We will now show that $x^{\prime}$ does not belong to a Type 1 cycle in the graph induced by $\mathcal{B}_{2} \cup \mathcal{P}_{e} \cup \mathcal{N}$. Suppose $x \in \mathcal{B}_{1}$. Since $\mathcal{N} \subseteq \mathcal{N}_{1}$, by Lemma 3, $x^{\prime}$ is adjacent to at least one vertex in $\mathcal{C} \cup \mathcal{P}_{i}$. Since $x \in \mathcal{B}_{1}$, $x^{\prime}$ cannot be adjacent to two vertices in $\mathcal{B}_{2} \cup \mathcal{P}_{e}$ and hence cannot belong to a Type 1 cycle. Now suppose $x \in \mathcal{P}_{2 e}$. If $x^{\prime}$ belongs to a Type 1 cycle, then it has two neighbors in $\mathcal{B}_{2} \cup \mathcal{P}_{e}$. By Lemma 7 , the remaining neighbor of $x^{\prime}$, that is $x$, does not belong to $\mathcal{P}_{2 e}$, which is a contradiction.

Thus, $x^{\prime}$ belongs to either a Type 2 cycle or a path in $\mathcal{B}_{2} \cup \mathcal{P}_{e} \cup \mathcal{N}$. From Observation 1312, $l\left(x^{\prime}, I_{1}\right)>n$ and therefore, $r\left(x, I_{1}\right)>n$. From the interval assignments for $x$ in (15) and (16), it immediately follows that $[0, n] \subset f\left(x, I_{1}\right)$.

\subsubsection{Vertices of $\mathcal{R}$}

$$
\forall v \in \mathcal{R}, f\left(v, I_{1}\right)=[-1, n] .
$$

Consider the set of vertices which have been assigned intervals until now: $\mathcal{A} \cup\left(\mathcal{B}_{2} \cup \mathcal{P}_{e} \cup \mathcal{N}\right) \cup\left(\mathcal{B}_{1} \cup \mathcal{P}_{2 e}\right) \cup \mathcal{R}=$ $V \backslash\left(\mathcal{P}_{2 i} \cup \mathcal{C}\right)$.

Lemma 11. $I_{1}\left[V \backslash\left(\mathcal{P}_{2 i} \cup \mathcal{C}\right)\right]$ is a supergraph of $G\left[V \backslash\left(\mathcal{P}_{2 i} \cup \mathcal{C}\right)\right]$.

Proof. Let $x, y \in V \backslash\left(\mathcal{P}_{2 i} \cup \mathcal{C}\right)$ be two adjacent vertices in $G$. If $x, y \in \mathcal{A} \cup \mathcal{B}_{2} \cup \mathcal{P}_{e} \cup \mathcal{N}$, then by Lemma 9 $x$ is adjacent to $y$ in $I_{1}$. Let $x \in \mathcal{B}_{1} \cup \mathcal{P}_{2 e} \cup \mathcal{R}$. By Lemma 10, $[0, n] \subset f\left(x, I_{1}\right)$.

If $y \in \mathcal{B}_{1} \cup \mathcal{P}_{2 e}$, again by Lemma 10, $[0, n] \subset f\left(y, I_{1}\right)$ and if $y \in \mathcal{R}$, then by (17), $[0, n] \subset f\left(y, I_{1}\right)$ and therefore, $x$ is adjacent to $y$. If $y \in \mathcal{A}$, then, since $\mathcal{B}_{1} \subseteq \mathcal{B}$ and $\mathcal{P}_{2 e} \subseteq \mathcal{P}$, by Table 1 (rows 1-3) $x$ cannot be adjacent to $y$ in $G$. Suppose $y \in \mathcal{B}_{2} \cup \mathcal{P}_{e}$. If $y$ belongs to a Type 1 cycle, then by Observation 1311(b), $f\left(y, I_{1}\right) \subset[1, n]$. Otherwise, from Observation 1313 $l\left(y, I_{1}\right)=n$. In either case, $x$ is adjacent to $y$ in $I_{1}$. Finally, suppose $y \in \mathcal{N}$. By Table 1 (row 2), $x \notin \mathcal{R}$, which implies that $x \in \mathcal{B}_{1} \cup \mathcal{P}_{2 e}$. From Table 2 (rows 2 and 3), $y$ is the unique neighbor of $x$ in $\mathcal{N}$. By (15) and (16), $r\left(x, I_{1}\right)=l\left(y, I_{1}\right)$ and therefore, $x$ and $y$ are adjacent in $I_{1}$. Hence proved.

\subsubsection{Vertices of $\mathcal{P}_{2 i}$}

Suppose $v \in \mathcal{P}_{2 i}$. Let $v^{\prime}$ be its unique neighbor in $\mathcal{R} \cup \mathcal{N}$ (see Table 2 row 4). Note that $f\left(v^{\prime}, I_{1}\right)$ is already defined Sections 3.1 .2 and 3.1.4.

$$
\begin{aligned}
& \text { if } v^{\prime} \in \mathcal{R} \text {, then, } f\left(v, I_{1}\right)=[-1,-1], \\
& \text { if } v^{\prime} \in \mathcal{N} \text {, then, } f\left(v, I_{1}\right)=\left[-1, l\left(v^{\prime}, I_{1}\right)\right] .
\end{aligned}
$$

Lemma 12. $I_{1}[V \backslash \mathcal{C}]$ is a supergraph of $G[V \backslash \mathcal{C}]$. 
Proof. Let $x, y \in V \backslash \mathcal{C}$ be two adjacent vertices in $G$. By Lemma 11, if $x, y \in V \backslash\left(\mathcal{C} \cup \mathcal{P}_{2 i}\right)$, then, they are adjacent in $I_{1}$. Suppose $x \in \mathcal{P}_{2 i}$. By definition, $x$ is an interior vertex of a special path in $\mathcal{P}$ and therefore, it is adjacent to two vertices from the path. Moreover, it is not adjacent to any end point of this path since otherwise it would be present in $\mathcal{P}_{2 e}$. Therefore, two of the neighbors of $x$ are in $\mathcal{P}_{2 i} \cup \mathcal{P}_{2 e}$. By Table 2 (row 4), the third neighbor of $x$ has to be in $\mathcal{R} \cup \mathcal{N}$. From this we infer that $y \in \mathcal{P}_{2 i} \cup \mathcal{P}_{2 e} \cup \mathcal{R} \cup \mathcal{N}$. Note that $l\left(x, I_{1}\right)=-1$ by (18) and (19). If $y \in \mathcal{P}_{2 i} \cup \mathcal{P}_{2 e} \cup \mathcal{R}$, then by the interval assignments (16 19), it follows that $l\left(y, I_{1}\right)=-1$. If $y \in \mathcal{N}$, then, by (19), $r\left(x, I_{1}\right)=l\left(y, I_{1}\right)$. In either case, $x$ is adjacent to $y$ in $I_{1}$. Hence proved.

Observation 14. If $x \in \mathcal{P}_{2 i}$ and $y \in \mathcal{R}$ are adjacent in $G$, then, $r\left(x, I_{1}\right)=l\left(y, I_{1}\right)$. From Table $\mathbf{Q}$ (row 4), $y$ is the only neighbor of $x$ in $\mathcal{R} \cup \mathcal{N}$. The interval assignment for $x$ is given by (18) and for $y$ by (17), from which it follows that $r\left(x, I_{1}\right)=l\left(y, I_{1}\right)=-1$.

\subsubsection{Vertices of $\mathcal{C}$}

We recall that $\mathcal{C}$ induces a collection of cycles in $G$.

Definition 9. Notation $\boldsymbol{\eta}(\cdot)$ and special vertex: We recall from Table 2 (row 3) that every vertex $x \in \mathcal{C}$ has a unique neighbor in $\mathcal{N}$. We denote this neighbor by $\eta(x)$. We define a vertex $c \in C$ as the special vertex of $C$ if $l\left(\eta(c), I_{1}\right)=\min _{c^{\prime} \in C} l\left(\eta\left(c^{\prime}\right), I_{1}\right)$. Note that $\eta(c)$ is already assigned an interval in Section 3.1.2.

Suppose $C$ is a cycle in $\mathcal{C}$. Let $C=c_{1} c_{2} \ldots c_{t} c_{1}$ be a natural ordering of the vertices of $C$ such that $c_{1}$ is the special vertex of $C$. The interval assignments are as follows:

$$
\begin{aligned}
& f\left(c_{1}, I_{1}\right)=\left[l\left(\eta\left(c_{1}\right), I_{1}\right), l\left(\eta\left(c_{1}\right), I_{1}\right)\right], \\
& f\left(c_{i}, I_{1}\right)= \begin{cases}{\left[l\left(\eta\left(c_{1}\right), I_{1}\right), l\left(\eta\left(c_{i}\right), I_{1}\right)+0.5\right],} & i=2, t, \\
\left.l l\left(\eta\left(c_{1}\right), I_{1}\right)+0.5, l\left(\eta\left(c_{i}\right), I_{1}\right)+0.5\right], & \text { otherwise. }\end{cases}
\end{aligned}
$$

Since $l\left(\eta\left(c_{1}\right), I_{1}\right)<l\left(\eta\left(c_{i}\right), I_{1}\right)$ for $i \neq 1$, we observe that the intervals are well-defined.

Observation 15. Let $C=c_{1} c_{2} \ldots c_{t} c_{1}$ be a cycle in $\mathcal{C}$ with $c_{1}$ being the special vertex.

1. $c_{1}$ is adjacent to only $c_{2}$ and $c_{t}$ in $I_{1}$. Further, $r\left(c_{1}, I_{1}\right)=l\left(c_{2}, I_{1}\right)=l\left(c_{t}, I_{1}\right)$. This is easy to infer from (20).

2. $C \backslash\left\{c_{1}\right\}$ is a clique in $I_{1}$. Since $l\left(\eta\left(c_{1}\right), I_{1}\right) \leq l\left(\eta(c), I_{1}\right), c \in C$, from (20), it follows that $\forall c \in C \backslash\left\{c_{1}\right\}$, $l\left(\eta\left(c_{1}\right)+0.5, I_{1}\right) \in f\left(c, I_{1}\right)$.

3. For every vertex $c \in \mathcal{C}, l\left(\eta(c), I_{1}\right) \in f\left(c, I_{1}\right)$ and therefore, $c$ is adjacent to $\eta(c)$ in $I_{1}$. The proof follows: Since $c_{1}$ is a special vertex, by definition, $l\left(\eta\left(c_{1}\right), I_{1}\right) \leq l\left(\eta(c), I_{1}\right)$, and therefore from the interval assignments in (20), $l\left(\eta(c), I_{1}\right) \in f\left(c, I_{1}\right)$.

Now we will show the following:

Lemma 13. $I_{1}$ is a supergraph of $G$.

Proof. Let $x, y \in V$ be two adjacent vertices in $G$. If $x, y \in V \backslash \mathcal{C}$, then, by Lemma 12, $x$ and $y$ are adjacent in $I_{1}$. If $x, y \in \mathcal{C}$, then, they belong to the same component (which is a special cycle) in $G[\mathcal{C}]$. From Observation 15 (Points 1 and 22), we can infer that they are adjacent in $I_{1}$. The only case remaining is the one in which one vertex is in $\mathcal{C}$ and the other in $V \backslash \mathcal{C}$.

Suppose $x \in \mathcal{C}$ and $y \in V \backslash \mathcal{C}$. Since $x$ belongs to a special cycle in $\mathcal{C}$, it has two neighbors in the cycle. The remaining neighbor is $y$. By Table 2 (row 3), $y \in \mathcal{N}$. Moreover, by the notation introduced in Definition 9. $y=\eta(x)$. From Observation [15I3, it follows that $y$ is adjacent to $x$ in $I_{1}$. Hence proved.

Recall that we need to show that $E(G)=E\left(I_{1}\right) \cap E\left(I_{2}\right) \cap E\left(I_{3}\right)$. For this, we will have to prove that every missing edge in $G$ is missing in at least one of the three interval graphs. Note that there is no edge between $\mathcal{A}$ and $V \backslash(\mathcal{A} \cup \mathcal{N}$ ) (Table 1 rows 1-3). Now we will show that all these missing edges of $G$ are also missing in $I_{1}$. 
Lemma 14. Let $x \in \mathcal{A}$ and $y \in V \backslash(\mathcal{A} \cup \mathcal{N})$. Then, $(x, y) \notin E\left(I_{1}\right)$.

Proof. From (13) $l\left(x, I_{1}\right)>2 n$. Now we will show that $r\left(y, I_{1}\right) \leq 2 n$, from which it immediately follows that $(x, y) \notin E\left(I_{1}\right)$. If $y \in \mathcal{B}_{2} \cup \mathcal{P}_{e}$, from Observations 1311 and 13]3, $r\left(y, I_{1}\right) \leq 2 n-1$. If $y \in \mathcal{B}_{1} \cup \mathcal{P}_{2 e}$, then by (15) and (16), $r\left(y, I_{1}\right)=l\left(z, I_{1}\right)$ for some $z \in \mathcal{N}$. If $y \in \mathcal{P}_{2 i}$, then by (18) and (19), either $r\left(y, I_{1}\right)=-1$ or $r\left(y, I_{1}\right)=l\left(z, I_{1}\right)$ for some $z \in \mathcal{N}$. If $y \in \mathcal{C}$, then from (20), $r\left(y, I_{1}\right) \leq l\left(z, I_{1}\right)+0.5$ for some $z \in \mathcal{N}$. If $y \in \mathcal{R}$, then by (17), $r\left(y, I_{1}\right)=n$. From Observations 131 and 132. $\forall z \in \mathcal{N}, l\left(z, I_{1}\right)<2 n-1$ and therefore, it follows that in each case $r\left(y, I_{1}\right)<2 n$. Thus, $(x, y) \notin E\left(I_{1}\right)$. Hence proved.

\subsection{Construction of $I_{2}$}

\subsubsection{Vertices of $\mathcal{A}$}

We recall the interval assignment for $\mathcal{A}$ in $I_{1}$ (see Section 3.1.1). Let $\overline{\Pi_{A}}$ be the reverse of $\Pi_{A}$. The interval assignments for vertices of $\mathcal{A}$ in $I_{2}$ are as follows:

An isolated vertex $u$ is given a point interval as follows:

$$
f\left(u, I_{2}\right)=\left[n+\overline{\Pi_{A}}(u), n+\overline{\Pi_{A}}(u)\right] .
$$

End points of an isolated edge $(u, v)$ : Without loss of generality, let $\Pi_{A}(v)=\Pi_{A}(u)+1$. This implies that $\overline{\Pi_{A}}(v)=\overline{\Pi_{A}}(u)-1$. We assign the intervals to $u$ and $v$ as follows:

$$
\begin{aligned}
& f\left(u, I_{2}\right)=\left[n+\overline{\Pi_{A}}(u)-0.5, n+\overline{\Pi_{A}}(u)\right], \\
& f\left(v, I_{2}\right)=\left[n+\overline{\Pi_{A}}(v), n+\overline{\Pi_{A}}(v)+0.5\right] .
\end{aligned}
$$

Note that the two intervals intersect at $n+\overline{\Pi_{A}}(u)-0.5=n+\overline{\Pi_{A}}(v)+0.5$.

Observation 16. The graph induced by $\mathcal{A}$ in $I_{2}$ and $G$ are identical, that is, $I_{2}[\mathcal{A}]=G[\mathcal{A}]$. The proof is similar to that of Lemma 8 .

\subsubsection{Vertices of $\mathcal{N}$}

Let $v \in \mathcal{N}$. From Table 2 (row 1 ), $v$ is adjacent to at most one vertex in $\mathcal{A}$.

$$
\begin{aligned}
& \text { if } v \text { is not adjacent to any vertex in } \mathcal{A} \text {, then, } f\left(v, I_{2}\right)=[0, n] \text {, } \\
& \text { if } v \text { is adjacent to vertex } a \text { in } \mathcal{A} \text {, then, } f\left(v, I_{2}\right)=\left[0, l\left(a, I_{2}\right)\right] .
\end{aligned}
$$

Note that $l\left(a, I_{2}\right)$ is already defined in (21) and (22) and satisfies, $l\left(a, I_{2}\right)>n$. Hence, we have the following observation.

Observation 17. In $I_{2}, \forall x \in \mathcal{N},[0, n] \subseteq f\left(x, I_{2}\right)$.

Lemma 15. $I_{2}[\mathcal{A} \cup \mathcal{N}]$ is a supergraph of $G[\mathcal{A} \cup \mathcal{N}]$.

Proof. Let $x, y \in \mathcal{A} \cup \mathcal{N}$ be two adjacent vertices in $G$. If $x, y \in \mathcal{A}$, from Observation 16, $(x, y) \in E\left(I_{2}\right)$. If $x, y \in \mathcal{N}$, from Observation 17, $(x, y) \in E\left(I_{2}\right)$. If $x \in \mathcal{N}$ and $y \in \mathcal{A}$, then by Table1(row 9) and Table 2 (row $1), x \in \mathcal{N}_{e}$ and $y$ is its only neighbor in $\mathcal{A}$. From (24), $r\left(x, I_{2}\right)=l\left(y, I_{2}\right)$ and therefore, $(x, y) \in E\left(I_{2}\right)$.

Lemma 16. If $x \in \mathcal{N}$ and $y \in \mathcal{A}$ such that $(x, y) \notin E(G)$, then, $(x, y) \notin E\left(I_{1} \cap I_{2}\right)$.

Proof. Suppose $x$ is not adjacent to any vertex in $\mathcal{A}$. In $I_{2}$, by (23), $r\left(x, I_{2}\right)=n$ and by (21) and (22), $l\left(y, I_{2}\right)>n$ and therefore, $(x, y) \notin I_{2}$. Let us assume that $x$ is adjacent to some vertex in $\mathcal{A}$, say $a$. From Table1 (row 9), $x \in \mathcal{N}_{e}$ and by Table 2 (row 1), $a$ is the only neighbor of $x$ in $\mathcal{A}$. From the interval assignment in (12), $r\left(x, I_{1}\right)=l\left(a, I_{1}\right)$ and from (24) $r\left(x, I_{2}\right)=l\left(a, I_{2}\right)$. If $\Pi_{A}(a)<\Pi_{A}(y)$, then, $l\left(a, I_{1}\right)<l\left(y, I_{1}\right)$ (this is easy to infer from (13) $)$ and therefore, $(x, y) \notin I_{1}$. Otherwise, since $\Pi_{A}(a)>\Pi_{A}(y)$, it implies that $\overline{\Pi_{A}}(a)<\overline{\Pi_{A}}(y)$ which in turn implies that $l\left(a, I_{2}\right)<l\left(y, I_{2}\right)$ (see interval assignments in (21) and (22)). Therefore, $(x, y) \notin E\left(I_{2}\right)$. 


\subsubsection{Vertices of $\mathcal{C} \cup \mathcal{P}$}

We recall that $\mathcal{C} \cup \mathcal{P}$ induces a collection of special cycles and special paths in $G$.

Definition 10. $\Pi_{2}$ is an ordering of $\mathcal{C} \cup \mathcal{P}$ such that the following properties are satisfied:

1. Let $P$ be a path in $\mathcal{P}$. For a natural ordering of $P$, say $p_{1} p_{2} \ldots p_{t}$, we have $\Pi_{2}\left(p_{i}\right)=\Pi_{2}\left(p_{i-1}\right)+1$, $2 \leq i \leq t$.

2. Suppose $C$ is a cycle in $\mathcal{C}$. For a natural ordering of $C$, say $c_{1} c_{2} \ldots c_{t} c_{1}$, where $c_{1}$ is the special vertex (recall Definition 9), we have $\Pi_{2}\left(c_{i}\right)=\Pi_{2}\left(c_{i-1}\right)+1,2 \leq i \leq t$.

It is easy to see that such an ordering $\Pi_{2}$ exists. Also note that if $S_{1}$ and $S_{2}$ are two different components of $G[\mathcal{C} \cup \mathcal{P}]$, then, either $\Pi_{2}\left(S_{1}\right)<\Pi_{2}\left(S_{2}\right)$ or $\Pi_{2}\left(S_{2}\right)<\Pi_{2}\left(S_{1}\right)$. The interval assignments are as follows:

For the vertices of a path: Suppose $P=p_{1} p_{2} \ldots p_{t}$ such that $\Pi_{2}\left(p_{i+1}\right)=\Pi_{2}\left(p_{i}\right)+1,1 \leq i<t$.

$$
\begin{aligned}
& f\left(p_{i}, I_{2}\right)=\left[\Pi_{2}\left(p_{i}\right), \Pi_{2}\left(p_{i}\right)+1\right], 1 \leq i<t, \\
& f\left(p_{t}, I_{2}\right)=\left[\Pi_{2}\left(p_{t}\right), \Pi_{2}\left(p_{t}\right)+0.5\right] .
\end{aligned}
$$

Observation 18. Let $P=p_{1} p_{2} \ldots p_{t}$ be a special path from $\mathcal{P}$ such that $\Pi_{2}\left(p_{i+1}\right)=\Pi_{2}\left(p_{i}\right)+1,1 \leq i<t$. Then, for $1<i \leq t, l\left(p_{i}, I_{2}\right)=r\left(p_{i-1}, I_{2}\right)$ and hence $I_{2}[P]=G[P]$.

For the vertices of a cycle: Suppose $C=c_{1} c_{2} \ldots c_{t} c_{1}$ such that $\Pi_{2}\left(c_{i+1}\right)=\Pi_{2}\left(c_{i}\right)+1,1 \leq i<t$.

$$
\begin{aligned}
& f\left(c_{1}, I_{2}\right)=\left[\Pi_{2}\left(c_{1}\right), \Pi_{2}\left(c_{t}\right)\right] \\
& f\left(c_{i}, I_{2}\right)=\left[\Pi_{2}\left(c_{i}\right), \Pi_{2}\left(c_{i}\right)+1\right], 1<i<t \\
& f\left(c_{t}, I_{2}\right)=\left[\Pi_{2}\left(c_{t}\right), \Pi_{2}\left(c_{t}\right)+0.5\right] .
\end{aligned}
$$

Observation 19. Suppose $C=c_{1} c_{2} \ldots c_{t} c_{1}$ is a special cycle from $\mathcal{C}$ such that $\Pi_{2}\left(c_{i+1}\right)=\Pi_{2}\left(c_{i}\right)+1$, $1 \leq i<t$.

1. For $2 \leq i \leq t, l\left(c_{i+1}, I_{2}\right)=r\left(c_{i}, I_{2}\right)$ and therefore, $I_{2}\left[C \backslash c_{1}\right]=G\left[C \backslash c_{1}\right]$.

2. $I_{2}[C]$ is a supergraph of $G[C]$. The proof is as follows: $c_{1}$ is adjacent to all the other vertices of $C$ in $I_{2}$ and from Point 1 in this observation, $I_{2}\left[C \backslash c_{1}\right]=G\left[C \backslash c_{1}\right]$.

Observation 20. For every $x \in \mathcal{C} \cup \mathcal{P}$ and $y \in \mathcal{N}, f\left(x, I_{2}\right) \subset f\left(y, I_{2}\right)$. The proof is as follows: $G$ is a cubic graph whereas $G[\mathcal{C} \cup \mathcal{P}]$ has maximum degree 2 and therefore, $\mathcal{C} \cup \mathcal{P} \neq V$. This implies that $\forall z \in \mathcal{C} \cup \mathcal{P}$, $\Pi_{2}(z)<n$. Taking this into consideration, from interval assignments (25) and (26) we can infer that $0<l\left(x, I_{2}\right)<r\left(x, I_{2}\right)<n$ and therefore, $f\left(x, I_{2}\right) \subset[0, n]$. From Observation $\left[17,[0, n] \subseteq f\left(y, I_{2}\right)\right.$. Hence proved.

Lemma 17. If $x, y \in \mathcal{C} \cup \mathcal{P}$ belong to different components in $G[\mathcal{C} \cup \mathcal{P}]$, then, $(x, y) \notin E\left(I_{2}\right)$.

Proof. Let $x \in S_{x}$ and $y \in S_{y}$, where $S_{x}$ and $S_{y}$ are two different components of $G[\mathcal{C} \cup \mathcal{P}]$. Without loss of generality we will assume that $\Pi_{2}\left(S_{x}\right)<\Pi_{2}\left(S_{y}\right)$. Irrespective of whether $S_{x}$ (or $S_{y}$ ) induces a path or a cycle in $G[\mathcal{C} \cup \mathcal{P}]$, from (25) and (26), it follows that $r\left(x, I_{2}\right) \leq \max _{a \in S_{x}} \Pi_{2}(a)+0.5<\min _{b \in S_{y}} \Pi_{2}(b) \leq l\left(y, I_{2}\right)$. Therefore, $(x, y) \notin E\left(I_{2}\right)$.

Lemma 18. The graph induced by $\mathcal{C} \cup \mathcal{P}$ in $G$ and $I_{1} \cap I_{2}$ are identical, that is, $G[\mathcal{C} \cup \mathcal{P}]=\left(I_{1} \cap I_{2}\right)[\mathcal{C} \cup \mathcal{P}]$.

Proof. Let $x, y \in \mathcal{C} \cup \mathcal{P}$. First we will show that if $(x, y) \in E(G)$, then $(x, y) \in E\left(I_{1} \cap I_{2}\right)$. Clearly, from Lemma 13, $(x, y) \in E\left(I_{1}\right)$. Since $x$ and $y$ are adjacent in $G$, they belong to the same path or cycle in $G[\mathcal{C} \cup \mathcal{P}]$, say $S$. From Observations 18 and 1922 it follows that $I_{2}[S]$ is a supergraph of $G[S]$. Therefore, $(x, y) \in E\left(I_{2}\right)$. Hence, $(x, y) \in E\left(I_{1} \cap I_{2}\right)$. 
$x_{1}$

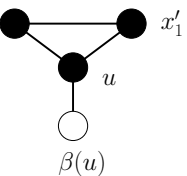

(a)

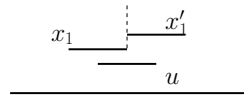

$\beta(u)$ $x_{1}$

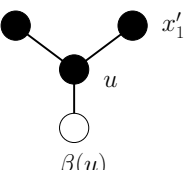

(b)

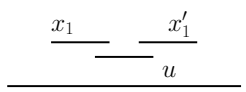

$\beta(u)$

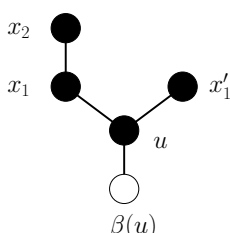

(c)

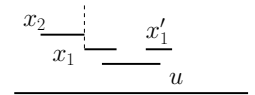

$\beta(u)$

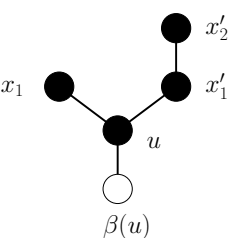

(d)

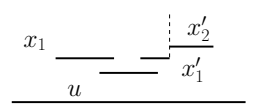

$\beta(u)$

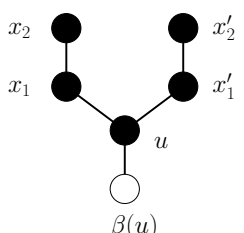

(e)

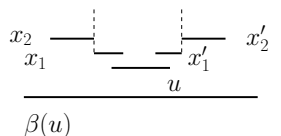

Fig. 3. The interval assignments for each component $\Gamma(u), u \in \mathcal{R}$ induced by $\mathcal{R} \cup \mathcal{B}$ in the interval graph $I_{2}$. The dotted vertical lines are used to indicate that the concerned intervals intersect exactly at their end points, that is, in (a) $r\left(x_{1}, I_{2}\right)=l\left(x_{1}^{\prime}, I_{2}\right)$, in (c) $r\left(x_{2}, I_{2}\right)=l\left(x_{1}, I_{2}\right)$, in (d) $l\left(x_{2}^{\prime}, I_{2}\right)=r\left(x_{1}^{\prime}, I_{2}\right)$ and in (e) $r\left(x_{2}, I_{2}\right)=l\left(x_{1}, I_{2}\right)$ and $l\left(x_{2}^{\prime}, I_{2}\right)=r\left(x_{1}^{\prime}, I_{2}\right)$.

Now we will show that if $(x, y) \notin E(G)$, then, either $(x, y) \notin E\left(I_{1}\right)$ or $(x, y) \notin E\left(I_{2}\right)$. There are two cases to consider: (1) $x$ and $y$ belong to different components in $G[\mathcal{C} \cup \mathcal{P}]$ and (2) they belong to the same component. If it is Case (1), then by Lemma 17. $(x, y) \notin E\left(I_{2}\right)$. If it is Case (2), then, let $x, y \in S$, where $S$ is a component of $G[\mathcal{C} \cup \mathcal{P}]$. If $S$ is a special path, then by Observation $18, I_{2}[S]=G[S]$ and therefore, $(x, y) \notin E\left(I_{2}\right)$. Suppose $S$ is a special cycle. Let $S=c_{1} c_{2} \ldots c_{t} c_{1}$, where $c_{1}$ is the special vertex. If neither $x$ nor $y$ is $c_{1}$, then, they are not adjacent in $I_{2}$. This is because, by Observation 1911 $I_{2}\left[C \backslash\left\{c_{1}\right\}\right]=G\left[C \backslash\left\{c_{1}\right\}\right]$. Suppose $x=c_{1}$, then clearly, $y \neq c_{2}, c_{t}$. By Observation [15], in $I_{1}, c_{1}$ is adjacent to only $c_{2}$ and $c_{t}$. Thus, $x$ and $y$ are not adjacent in $I_{1}$. Hence, $(x, y) \notin E\left(I_{1} \cap I_{2}\right)$.

Lemma 19. $I_{2}[\mathcal{A} \cup \mathcal{N} \cup \mathcal{C} \cup \mathcal{P}]$ is a super graph of $G[\mathcal{A} \cup \mathcal{N} \cup \mathcal{C} \cup \mathcal{P}]$.

Proof. Let $x, y \in \mathcal{A} \cup \mathcal{N} \cup \mathcal{C} \cup \mathcal{P}$ be two adjacent vertices in $G$. If $x, y \in \mathcal{A} \cup \mathcal{N}$, then by Lemma 15, $(x, y) \in E\left(I_{2}\right)$ and if $x, y \in \mathcal{C} \cup \mathcal{P}$, then, from Lemma 18 we can infer that $(x, y) \in E\left(I_{2}\right)$. Therefore, we will assume that $x \in \mathcal{A} \cup \mathcal{N}$ and $y \in \mathcal{C} \cup \mathcal{P}$. By Table 1 (row 1), $x \notin \mathcal{A}$ and hence, $x \in \mathcal{N}$. From Observation 20, $f\left(y, I_{2}\right) \subset f\left(x, I_{2}\right)$. Therefore, $(x, y) \in E\left(I_{2}\right)$.

\subsubsection{Vertices of $\mathcal{R} \cup \mathcal{B}$}

From Lemma 4, we recall that each component in $\mathcal{R} \cup \mathcal{B}$ is isomorphic to one of the graphs shown in Figure 1 Further, each component contains exactly one vertex from $\mathcal{R}$ and is uniquely identified by it; by the notation introduced in Lemma 4, for every $u \in \mathcal{R}, \Gamma(u)$ denotes the component containing $u$ in $G[\mathcal{R} \cup \mathcal{B}]$.

Definition 11. Notation $\boldsymbol{\beta}(\cdot)$ : In the graph induced by $\mathcal{R} \cup \mathcal{B}$, consider each component $\Gamma(u), u \in \mathcal{R}$. From Table $\mathbf{Q}$ (row 5), $u$ is adjacent to a unique vertex in $\mathcal{P}_{2 i}$. We denote this vertex by $\beta(u)$.

Interval assignments for vertices of $\mathcal{R} \cup \mathcal{B}$ : Let us consider a component of $G[\mathcal{R} \cup \mathcal{B}]$, say $\Gamma(u), u \in \mathcal{R}$. From (25), we note that $\beta(u)$ is assigned a unit interval in $I_{2}$. The interval assignments for the vertices of $\Gamma(u)$ is illustrated in Figure 3 .

Remark 1. Let $u \in \mathcal{R}$. Every vertex of $\Gamma(u)$ is assigned a strict sub-interval of $f\left(\beta(u), I_{2}\right)$ and none of these intervals contains any end point of $f\left(\beta(u), I_{2}\right)$.

Lemma 20. Let $z \in \mathcal{R}$ and $\Gamma(z)$ be a component of $G[\mathcal{R} \cup \mathcal{B}]$.

1. Every vertex in $\Gamma(z)$ is adjacent to only one vertex in $\mathcal{C} \cup \mathcal{P}$ and that is $\beta(z)$.

2. The graph induced by $\mathcal{R} \cup \mathcal{B}$ in $I_{2}$ and in $G$ are identical, that is, $I_{2}[\mathcal{R} \cup \mathcal{B}]=G[\mathcal{R} \cup \mathcal{B}]$. 
Proof. Consider the vertex $\beta(z)$. Let $f_{o}\left(\beta(z), I_{2}\right)$ denote the open interval $\left(l\left(\beta(z), I_{2}\right), r\left(\beta(z), I_{2}\right)\right)$. From Definition [1], we recall that $\beta(z) \in \mathcal{P}_{2 i}$. We first prove the following:

Claim 1. Let $p \in \mathcal{C} \cup \mathcal{P}$ such that $p \neq \beta(z)$. Then, $f_{o}\left(\beta(z), I_{2}\right) \cap f\left(p, I_{2}\right)=\varnothing$.

Proof. If $\beta(z)$ and $p$ belong to different components in $G[\mathcal{C} \cup \mathcal{P}]$, then by Lemma 17, $(\beta(z), p) \notin E\left(I_{2}\right)$ and therefore, their intervals do not intersect. Suppose $\beta(z)$ and $p$ are in the same component. Since $\beta(z) \in \mathcal{P}_{2 i}$, $\beta(z)$ and $p$ belong to a special path. By (25), it follows that $f\left(\beta(z), I_{2}\right)$ and $f\left(p, I_{2}\right)$ can intersect only at $l\left(\beta(z), I_{2}\right)$ or $r\left(\beta(z), I_{2}\right)$. Hence proved.

By Remark 1 for every $x \in \Gamma(z), f\left(x, I_{2}\right) \subseteq f_{o}\left(\beta(z), I_{2}\right)$. This implies that $x$ is adjacent to $\beta(z)$ in $I_{2}$ and by Claim 1, $x$ is not adjacent to any other vertex from $\mathcal{C} \cup \mathcal{P}$. Thus, we have proved the first statement.

Suppose $x \in \Gamma(z)$ and $y \in \Gamma\left(z^{\prime}\right)$, where $z \neq z^{\prime}$. We first note that $\beta(z) \neq \beta\left(z^{\prime}\right)$. This is because, since $\beta(z) \in \mathcal{P}_{2 i}$, in $G[\mathcal{C} \cup \mathcal{P}]$, it is the interior vertex of a special path and therefore, two of its neighbors belong to $\mathcal{P}$. Since its remaining neighbor is $z$, it cannot be adjacent to $z^{\prime}$. By Remark $1, f\left(x, I_{2}\right) \subseteq f_{o}\left(\beta(z), I_{2}\right)$ and $f\left(y, I_{2}\right) \subseteq f_{o}\left(\beta\left(z^{\prime}\right), I_{2}\right)$. From Claim 1, $f_{o}\left(\beta(z), I_{2}\right) \cap f_{o}\left(\beta\left(z^{\prime}\right), I_{2}\right)=\varnothing$ and therefore, $x$ is not adjacent to $y$. From Figure 3, it is easy to see that $I_{2}[\Gamma(z)]=G[\Gamma(z)] \forall z \in \mathcal{R}$. Therefore, $I_{2}[\mathcal{R} \cup \mathcal{B}]=G[\mathcal{R} \cup \mathcal{B}]$.

Observation 21. Here are some immediate consequences of Lemma 20.

1. If $x \in \mathcal{R}$ and $y \in \mathcal{P}_{2 i}$ such that $(x, y) \notin E(G)$, then, $(x, y) \notin E\left(I_{2}\right)$. This follows from noting that $y \neq \beta(x)$ and subsequently applying Lemma 20 (Statement 1).

2. For any $x \in \mathcal{R} \cup \mathcal{B}$ and $y \in \mathcal{C} \cup \mathcal{P}_{e} \cup \mathcal{P}_{2 e},(x, y) \notin E\left(I_{2}\right)$. The proof is as follows: Let $x \in \Gamma(z), z \in \mathcal{R}$. From Lemma 20] (Statement 1), $x$ is not adjacent to any vertex in $\mathcal{C} \cup \mathcal{P}$ other than $\beta(z)$ in $I_{2}$. From Definition 11, $\beta(z) \in \mathcal{P}_{2 i}$ and therefore, $y \neq \beta(z)$.

Lemma 21. $I_{2}$ is a supergraph of $G$.

Proof. Let $x$ and $y$ be two adjacent vertices in $G$. If $x, y \in \mathcal{A} \cup \mathcal{N} \cup \mathcal{C} \cup \mathcal{P}$, then, by Lemma 19, $(x, y) \in E\left(I_{2}\right)$. If $x, y \in \mathcal{R} \cup \mathcal{B}$, then, by Lemma 20 (Statement 2), $(x, y) \in E\left(I_{2}\right)$. The only remaining case is when $x \in \mathcal{A} \cup \mathcal{N} \cup \mathcal{C} \cup \mathcal{P}$ and $y \in \mathcal{R} \cup \mathcal{B}$. Let $\Gamma(z), z \in \mathcal{R}$ be the component in $G[\mathcal{R} \cup \mathcal{B}]$ containing $y$. By Table 1 (rows 2, 3, 5 and 6 ), $x \notin \mathcal{A} \cup \mathcal{C}$ and therefore, $x \in \mathcal{P} \cup \mathcal{N}$.

Suppose $x \in \mathcal{P}$. By Table 1 (row 6), $y \notin \mathcal{B}$. Since by assumption $y \in \mathcal{R} \cup \mathcal{B}$, it implies that $y \in \mathcal{R}$, which in turn implies that $z=y$ as $z$ is the only vertex from $\mathcal{R}$ in $\Gamma(z)$. From Table 1 (row 5 ), we can infer that $x \in \mathcal{P}_{2 i}$. Now by Definition [1], $x=\beta(y)$. By Lemma [20 (Statement 1), $y$ is adjacent to $x$ in $I_{2}$. Finally, if $x \in \mathcal{N}$, then by Observation 20, $f\left(x, I_{2}\right) \supset f\left(\beta(z), I_{2}\right)$, since $\beta(z) \in \mathcal{P}_{2 i} \subset \mathcal{P}$. By Remark 1 $f\left(y, I_{2}\right) \subset f\left(\beta(z), I_{2}\right)$. Therefore, $f\left(y, I_{2}\right) \subset f\left(x, I_{2}\right)$ and $x$ is adjacent to $y$ in $I_{2}$.

\subsection{Construction of $I_{3}$}

\subsubsection{Vertices of $\mathcal{B}_{2} \cup \mathcal{P}_{e} \cup \mathcal{N}$}

We recall the notations and interval assignments developed for this set in $I_{1}$, in particular the definition of $\Pi_{1}$ (Definition 8 ).

For a vertex in a Type 1 cycle: Let $S=c_{1} c_{2} \ldots c_{t} c_{1}$ be a Type 1 cycle such that $\Pi_{1}\left(c_{i+1}\right)=\Pi_{1}\left(c_{i}\right)+1$, $1 \leq i<t$. We recall that $c_{1} \in \mathcal{N}$ and $c_{t} \in \mathcal{B}_{2} \cup \mathcal{P}_{e}$. The interval assignments are as follows: For $1 \leq i<t$,

$$
\begin{aligned}
& \text { if } c_{i} \in \mathcal{N}, \text { then, } f\left(c_{i}, I_{3}\right)=\left[\Pi_{1}\left(c_{i}\right), \Pi_{1}\left(c_{i}\right)+1\right], \\
& \text { if } c_{i} \in \mathcal{B}_{2}, \text { then, } f\left(c_{i}, I_{3}\right)=\left[\Pi_{1}\left(c_{i}\right), n\right], \\
& \text { if } c_{i} \in \mathcal{P}_{e}, \text { then, } f\left(c_{i}, I_{3}\right)=\left[\Pi_{1}\left(c_{i}\right), n+1\right] .
\end{aligned}
$$

The interval assignment for $c_{t}$ is as follows:

$$
\begin{aligned}
& \text { if } c_{t} \in \mathcal{B}_{2}, \text { then, } f\left(c_{t}, I_{3}\right)=\left[\Pi_{1}\left(c_{1}\right)+1, n\right], \\
& \text { if } c_{t} \in \mathcal{P}_{e}, \text { then, } f\left(c_{t}, I_{3}\right)=\left[\Pi_{1}\left(c_{1}\right)+1, n+1\right],
\end{aligned}
$$


Observation 22. Consider a Type 1 cycle $S=c_{1} c_{2} \ldots c_{t} c_{1}$, such that $\Pi_{1}\left(c_{i+1}\right)=\Pi_{1}\left(c_{i}\right)+1,1 \leq i<t$.

1. $I_{3}[S]$ is a supergraph of $G[S]$. The proof is as follows: From Observation 9, $\Pi_{1}\left(c_{i}\right)<n-1$. Using this in (2729), we note that for $1 \leq i<t, l\left(c_{i}, I_{3}\right)=\Pi_{1}\left(c_{i}\right)$ and $\Pi_{1}\left(c_{i}\right)+1 \in f\left(c_{i}, I_{3}\right)$, and therefore, $c_{i}$ is adjacent to $c_{i+1}$ in $I_{3}$. Next, from (30) and (31), it is easy to infer that $f\left(c_{t}, I_{3}\right)$ contains $\Pi_{1}\left(c_{i}\right)+1$, $1 \leq i \leq t$. Therefore, $c_{t}$ is adjacent to all the other vertices of the cycle. Hence proved.

2. $r\left(c_{1}, I_{3}\right)=l\left(c_{2}, I_{3}\right)=l\left(c_{t}, I_{3}\right)$.

Lemma 22. Let $x \in \mathcal{N}$ and $y \in \mathcal{B}_{2} \cup \mathcal{P}_{e} \cup \mathcal{N}$ be such that $x \in S_{x}$ and $y \in S_{y}$ where both $S_{x}$ and $S_{y}$ induce Type 1 cycles. If $(x, y) \notin E(G)$, then $(x, y) \notin E\left(I_{1} \cap I_{3}\right)$.

Proof. If $S_{x} \neq S_{y}$, by Observation 1013. $(x, y) \notin E\left(I_{1}\right)$. Suppose $S_{x}=S_{y}=S$. Let $S=c_{1} c_{2} \ldots c_{t} c_{1}$ such that $\Pi_{1}\left(c_{i+1}\right)=\Pi_{1}\left(c_{i}\right)+1,1 \leq i<t$. If neither $x$ nor $y$ is $c_{1}$, then by Observation 101, they are not adjacent in $I_{1}$ since $I_{1}\left[S \backslash\left\{c_{1}\right\}\right]=G\left[S \backslash\left\{c_{1}\right\}\right]$. If $x=c_{1}$, then, $y=c_{j}$ for some $j \neq 2, t$. In $I_{3}$, noting that $c_{1} \in \mathcal{N}$, from (27), $r\left(x, I_{3}\right)=r\left(c_{1}, I_{3}\right)=\Pi_{1}\left(c_{1}\right)+1$. From (27) 29$) l\left(y, I_{3}\right)=\Pi_{1}(y)=\Pi_{1}\left(c_{j}\right)>\Pi_{1}\left(c_{1}\right)+1$. Hence, $(x, y) \notin E\left(I_{3}\right)$.

For a vertex in a Type 2 cycle: Let $S=c_{1} c_{2} \ldots c_{t} c_{1}$ be a Type 2 cycle such that $\Pi_{1}\left(c_{i+1}\right)=\Pi_{1}\left(c_{i}\right)+1$, $1 \leq i<t$. We recall from Definition 8 (Point 2) that $c_{1}, c_{t} \in \mathcal{N}$. They are assigned intervals as follows:

$$
\begin{aligned}
& f\left(c_{1}, I_{3}\right)=\left[\Pi_{1}\left(c_{1}\right), \Pi_{1}\left(c_{1}\right)+1\right], \\
& f\left(c_{t}, I_{3}\right)=\left[\Pi_{1}\left(c_{1}\right)+1, \Pi_{1}\left(c_{t}\right)+0.5\right],
\end{aligned}
$$

For $c_{i}, 1<i<t$,

$$
\begin{aligned}
& \text { if } c_{i} \in \mathcal{N} \text {, then, } f\left(c_{i}, I_{3}\right)=\left[\Pi_{1}\left(c_{i}\right), \Pi_{1}\left(c_{i}\right)+1\right], \\
& \text { if } c_{i} \in \mathcal{B}_{2}, \text { then, } f\left(c_{i}, I_{3}\right)=\left[\Pi_{1}\left(c_{i}\right), n\right] \\
& \text { if } c_{i} \in \mathcal{P}_{e}, \text { then, } f\left(c_{i}, I_{3}\right)=\left[\Pi_{1}\left(c_{i}\right), n+1\right] .
\end{aligned}
$$

Observation 23. Let $S=c_{1} c_{2} \ldots c_{t} c_{1}$ be a Type 2 cycle such that $\Pi_{1}\left(c_{i+1}\right)=\Pi_{1}\left(c_{i}\right)+1,1 \leq i<t$.

1. $I_{3}[S]$ is a supergraph of $G[S]$. The proof is as follows: Recalling from Observation 9 that $\Pi_{1}(z)<n-1$, $\forall z \in \mathcal{B}_{2} \cup \mathcal{P}_{e} \cup \mathcal{N}$, from (32) and (34 36]), for $1 \leq i<t, \Pi_{1}\left(c_{i}\right), \Pi_{1}\left(c_{i}\right)+1 \in f\left(c_{i}, I_{3}\right)$. Therefore, $c_{i}$ is adjacent to $c_{i+1}, 1 \leq i<t$. From (33), for $1 \leq i<t, \Pi_{1}\left(c_{i}\right)+1 \in f\left(c_{t}, I_{3}\right)$. Therefore, $c_{t}$ is adjacent to all the other vertices of $S$. Hence proved.

2. $r\left(c_{1}, I_{3}\right)=l\left(c_{2}, I_{3}\right)=l\left(c_{t}, I_{3}\right)$.

3. Let $x, y \in S$ be two adjacent vertices in $G$ such that neither $x$ nor $y$ is $c_{t}$. If $\Pi_{1}(x)<\Pi_{1}(y)$ and $x \in \mathcal{N}$, then, $r\left(x, I_{3}\right)=l\left(y, I_{3}\right)$. This follows by noting that $\Pi_{1}(y)=\Pi_{1}(x)+1$ and subsequently applying it in (32) and $34(36)$.

For a vertex in a path: Let $S=p_{1} p_{2} \ldots p_{t}$ be a path such that $\Pi_{1}\left(p_{i+1}\right)=\Pi_{1}\left(p_{i}\right)+1,1 \leq i<t$. We recall from Observation 612 and Definition 5 that $p_{1}, p_{t} \in \mathcal{N}_{e}$. The interval assignment for $p_{t}$ is as follows:

$$
f\left(p_{t}, I_{3}\right)=\left[\Pi_{1}\left(p_{t}\right), \Pi_{1}\left(p_{t}\right)+0.5\right] .
$$

The interval assignment for $p_{i}, i<t$ is as follows:

$$
\begin{aligned}
& \text { if } p_{i} \in \mathcal{N}, \text { then, } f\left(p_{i}, I_{3}\right)=\left[\Pi_{1}\left(p_{i}\right), \Pi_{1}\left(p_{i}\right)+1\right], \\
& \text { if } p_{i} \in \mathcal{B}_{2}, \text { then, } f\left(p_{i}, I_{3}\right)=\left[\Pi_{1}\left(p_{i}\right), n\right], \\
& \text { if } p_{i} \in \mathcal{P}_{e}, \text { then, } f\left(p_{i}, I_{3}\right)=\left[\Pi_{1}\left(p_{i}\right), n+1\right] .
\end{aligned}
$$

Observation 24. Let $S=p_{1} p_{2} \ldots p_{t}$ be a path such that $\Pi_{1}\left(p_{i+1}\right)=\Pi_{1}\left(p_{i}\right)+1,1 \leq i<t$. 
1. $I_{3}[S]$ is a supergraph of $G[S]$. The proof is as follows: Since by Observation 9, $\Pi_{1}\left(p_{i}\right)<n-1$, we can infer from the interval assignments in (38-40) that, for $i<t$, the points $\Pi_{1}\left(p_{i}\right)$ and $\Pi_{1}\left(p_{i}\right)+1$ belong to $f\left(p_{i}, I_{3}\right)$. From (37), the point $\Pi_{1}\left(p_{t}\right)$ belongs to $f\left(p_{t}, I_{3}\right)$. This implies that for $1 \leq i<t, p_{i}$ is adjacent to $p_{i+1}$. Hence, proved.

2. Let $x, y \in S$ be two adjacent vertices in $G$. If $\Pi_{1}(x)<\Pi_{1}(y), x \in \mathcal{N}$ and $y \in \mathcal{B}_{2} \cup \mathcal{P}_{e} \cup \mathcal{N}$, then, $r\left(x, I_{3}\right)=l\left(y, I_{3}\right)$. This follows by noting that $\Pi_{1}(y)=\Pi_{1}(x)+1$ and subsequently applying it in 37 40).

Observation 25. Here are some observations regarding the intervals assigned to vertices of $\mathcal{B}_{2} \cup \mathcal{P}_{e} \cup \mathcal{N}$. We recall from Observation 9 that for any $z \in \mathcal{B}_{2} \cup \mathcal{P}_{e} \cup \mathcal{N}, \Pi_{1}(z)<n-1$.

1. If $z \in \mathcal{N}, \Pi_{1}(z)+0.5 \leq r\left(z, I_{3}\right) \leq \Pi_{1}(z)+1<n$. This follows from the interval assignments in (27), (32 344 and $37(38)$.

2. If $z \in \mathcal{B}_{2} \cup \mathcal{P}_{e}$ belongs to a Type 2 cycle or a path, then, $l\left(z, I_{3}\right)=\Pi_{1}(z)<n-1$. This follows from (35 36) for Type 2 cycles and (39 40) for paths respectively.

3. If $z \in \mathcal{B}_{2}$, from (28), (30), (35) and (39), $r\left(x, I_{3}\right)=n$.

4. If $z \in \mathcal{P}_{e}$, from (29), (31), (36) and (40), $r\left(x, I_{3}\right)=n+1$.

Observation 26. $I_{3}\left[\mathcal{B}_{2} \cup \mathcal{P}_{e} \cup \mathcal{N}\right]$ is a supergraph of $G\left[\mathcal{B}_{2} \cup \mathcal{P}_{e} \cup \mathcal{N}\right]$. The proof is as follows: Let $S$ be a component of $G\left[\mathcal{B}_{2} \cup \mathcal{P}_{e} \cup \mathcal{N}\right]$. It is either a Type 1 cycle, Type 2 cycle or path. From Observations [2211, 23:1] and 241, it follows that $I_{3}[S]$ is a supergraph of $G[S]$.

Lemma 23. Let $x \in \mathcal{N}$ and $y \in \mathcal{B}_{2} \cup \mathcal{P}_{e} \cup \mathcal{N}$ be such that $x \in S_{x}$ and $y \in S_{y}$ where $S_{x}$ and $S_{y}$ induce a Type 2 cycle or a path. If $(x, y) \notin E(G)$, then $(x, y) \notin E\left(I_{1} \cap I_{3}\right)$.

Proof. We will consider the following two cases separately: (1) $y \in \mathcal{N}$ and (2) $y \in \mathcal{B}_{2} \cup \mathcal{P}_{e}$.

$\boldsymbol{y} \in \mathcal{N}$ : If $S_{x} \neq S_{y}$, then, $(x, y) \notin E\left(I_{3}\right)$. The proof is as follows: Without loss of generality let $\Pi_{1}\left(S_{x}\right)<$ $\Pi_{1}\left(S_{y}\right)$. From the interval assignments for a vertex of $\mathcal{N}$ in a Type 2 cycle (see (32 34) ) and a path (see (37] 38) $), r\left(x, I_{3}\right) \leq \max _{a \in S_{x}} \Pi_{1}(a)+0.5<\min _{b \in S_{y}} \Pi_{1}(b) \leq l\left(y, I_{3}\right)$ and therefore, $(x, y) \notin E\left(I_{3}\right)$.

Now we consider the case $S_{x}=S_{y}=S$. Let $S=s_{1} s_{2} \ldots s_{t}$, such that $\Pi_{1}\left(s_{i+1}\right)=\Pi_{1}\left(s_{i}\right)+1$, for $1 \leq i \leq t$. Since $x$ and $y$ are not adjacent in $S$, by the definition of $\Pi_{1}$ (Definition 8 , Points 1 and 2 for paths and Type 2 cycles respectively), $\left|\Pi_{1}(x)-\Pi_{1}(y)\right|>1$. Suppose $S$ is a Type 2 cycle. If neither $x$ nor $y$ is $s_{t}$, then, by (32) and (34), $f\left(x, I_{3}\right)=\left[\Pi_{1}(x), \Pi_{1}(x)+1\right]$ and $f\left(y, I_{3}\right)=\left[\Pi_{1}(y), \Pi_{1}(y)+1\right]$. Since $\left|\Pi_{1}(x)-\Pi_{1}(y)\right|>1$, $f\left(x, I_{3}\right) \cap f\left(y, I_{3}\right)=\varnothing$. If $x=s_{t}$, then, in $I_{1}$, from (8), $l\left(x, I_{1}\right)=n+\Pi_{1}\left(s_{t}\right)$ while, since $y \neq s_{t-1}, s_{1}$ from (9), $r\left(y, I_{1}\right)=n+\Pi_{1}(y)+1<n+\Pi_{1}\left(s_{t}\right)=l\left(x, I_{1}\right)$. Therefore, $(x, y) \notin E\left(I_{1}\right)$. If $S$ is a path, then, assuming without loss of generality that $\Pi_{1}(x)<\Pi_{1}(y)$, from (37) and (38), $r\left(x, I_{3}\right)=\Pi_{1}(x)+1$ and $l\left(y, I_{3}\right)=\Pi_{1}(y)$. Since $\left|\Pi_{1}(x)-\Pi_{1}(y)\right|>1, r\left(x, I_{3}\right)<l\left(y, I_{3}\right)$. Therefore, $(x, y) \notin E\left(I_{3}\right)$.

$\boldsymbol{y} \in \mathcal{B}_{\mathbf{2}} \cup \mathcal{P}_{\boldsymbol{e}}$ : First we will show the following:

Claim 2. $\left|\Pi_{1}(x)-\Pi_{1}(y)\right|>1$.

Proof. Suppose $S_{x}=S_{y}$, that is, both $x$ and $y$ belong to the same component. Since $x$ is not adjacent to $y$, from the definition of $\Pi_{1}$ (Definition 8), $\left|\Pi_{1}(x)-\Pi_{1}(y)\right|>1$. Suppose $S_{x} \neq S_{y}$. Let $S_{y}=s_{1} s_{2} \ldots s_{t}$ such that $\Pi_{1}\left(s_{i+1}\right)=\Pi_{1}\left(s_{i}\right)+1,1 \leq i<t$. If $\left|\Pi_{1}(x)-\Pi_{1}(y)\right|=1$, then, $y$ must be either $s_{1}$ or $s_{t}$. But, $s_{1}, s_{t} \in \mathcal{N}$ since $S_{y}$ is either a Type 2 cycle or a path (by Definition 8 ). This contradicts the assumption that $y \in \mathcal{B}_{2} \cup \mathcal{P}_{e}$.

Suppose $\Pi_{1}(x)<\Pi_{1}(y)$. From Observation 25!1 $r\left(x, I_{3}\right) \leq \Pi_{1}(x)+1$ and from Observation [2512 $l\left(y, I_{3}\right)=\Pi_{1}(y)$. We have from Claim[2, $\Pi_{1}(x)+1<\Pi_{1}(y)$ and hence $(x, y) \notin E\left(I_{3}\right)$. Suppose $\Pi_{1}(x)>\Pi_{1}(y)$. From Observation 1313, $r\left(y, I_{1}\right)=n+\Pi_{1}(y)+1$ and from Observation 132, $l\left(x, I_{1}\right)=n+\Pi_{1}(x)$. From Claim 2, $\Pi_{1}(y)+1<\Pi_{1}(x)$ and hence $(x, y) \notin E\left(I_{1}\right)$.

Observation 27. If $x \in \mathcal{N}$ and $y \in \mathcal{B}_{2} \cup \mathcal{P}_{e} \cup \mathcal{N}$ such that $(x, y) \notin E(G)$, then, $(x, y) \notin E\left(I_{1} \cap I_{3}\right)$. The proof is as follows: If one of $x$ and $y$ belongs to a Type 1 cycle and the other belongs to a Type 2 cycle or path, from Observation 135, $(x, y) \notin E\left(I_{1}\right)$. If both $x$ and $y$ belong to Type 1 cycles, then, by Lemma 22, $(x, y) \notin E\left(I_{1} \cap I_{3}\right)$. If both $x$ and $y$ belong to Type 2 cycles and paths, then, by Lemma 23. $(x, y) \notin E\left(I_{1} \cap I_{3}\right)$. 


\subsubsection{Vertices of $\mathcal{B}_{1} \cup \mathcal{C} \cup \mathcal{P}_{2 e}$}

Let $v \in \mathcal{B}_{1} \cup \mathcal{C} \cup \mathcal{P}_{2 e}$. By Table2 (rows 2 and 3), it follows that $v$ is adjacent to exactly one vertex in $\mathcal{N}$; let $v^{\prime}$ be this vertex.

$$
\begin{aligned}
& \text { If } v \in \mathcal{C} \cup \mathcal{P}_{2 e} \text {, then, } f\left(v, I_{3}\right)=\left[r\left(v^{\prime}, I_{3}\right), n+1\right] \text {, } \\
& \text { If } v \in \mathcal{B}_{1} \text {, then, } f\left(v, I_{3}\right)=\left[r\left(v^{\prime}, I_{3}\right), n\right],
\end{aligned}
$$

Note that $v^{\prime}$ is already assigned an interval in Section 3.3.1.

\subsubsection{Vertices of $\mathcal{P}_{2 i}$}

Let $v \in \mathcal{P}_{2 i}$. By Table 2 (row 4 ), $v$ has a unique neighbor in $\mathcal{R} \cup \mathcal{N}$; let $v^{\prime}$ be this vertex.

$$
\begin{aligned}
& \text { if } v^{\prime} \in \mathcal{R}, \text { then, } f\left(v, I_{3}\right)=[n+1, n+1], \\
& \text { if } v^{\prime} \in \mathcal{N}, \text { then, } f\left(v, I_{3}\right)=\left[r\left(v^{\prime}, I_{3}\right), n+1\right] .
\end{aligned}
$$

Lemma 24. Let $x \in \mathcal{P}_{2 i}$ and $y \in \mathcal{B}$. $(x, y) \notin E\left(I_{2} \cap I_{3}\right)$.

Proof. Recall the notation $\beta(\cdot)$ introduced in Definition 11 Let $y \in \Gamma(z)$ for some $z \in \mathcal{R}$. If $x \neq \beta(z)$, then by Lemma 20 (Point 1$),(x, y) \notin E\left(I_{2}\right)$. If $x=\beta(z)$, then, it implies that $x$ is adjacent to $z$ and by Table 2 (row 4), $z$ is its only neighbor in $\mathcal{R} \cup \mathcal{N}$. Since $z \in \mathcal{R}$, the interval assigned to $x$ is given in (43), from which $l\left(x, I_{3}\right)=n+1$. If $y \in \mathcal{B}_{1}$, then by (42), $r\left(y, I_{3}\right)=n$. If $y \in \mathcal{B}_{2}$, from Observation 25]3, $r\left(y, I_{3}\right)=n$. Therefore, $(x, y) \notin E\left(I_{3}\right)$.

\subsubsection{Vertices of $\mathcal{R}$}

Every vertex is assigned the following interval:

$$
\forall v \in \mathcal{R}, f\left(v, I_{3}\right)=[n, n+1] .
$$

Lemma 25. $I_{3}\left[\mathcal{B}_{1} \cup \mathcal{C} \cup \mathcal{P}_{2 e} \cup \mathcal{P}_{2 i} \cup \mathcal{R}\right]$ is a supergraph of $G\left[\mathcal{B}_{1} \cup \mathcal{C} \cup \mathcal{P}_{2 e} \cup \mathcal{P}_{2 i} \cup \mathcal{R}\right]$.

Proof. First we prove the following:

Claim 3. For any $x \in \mathcal{C} \cup \mathcal{P}_{2 e} \cup \mathcal{R},[n, n+1] \subseteq f\left(x, I_{3}\right)$.

Proof. For any $z \in \mathcal{N}$, from Observation 2511, $r\left(z, I_{3}\right) \leq \Pi_{1}(z)+1<n$. From (41), for every $x \in \mathcal{C} \cup \mathcal{P}_{2 e}$, $l\left(x, I_{3}\right)=r\left(z, I_{3}\right)$ for some $z \in \mathcal{N}$. Since $r\left(x, I_{3}\right)=n+1$, it implies that $[n, n+1] \subset f\left(x, I_{3}\right)$. If $x \in \mathcal{R}$, by (45), $[n, n+1]=f\left(x, I_{3}\right)$. Hence proved.

To prove the lemma, we need to show that if $(x, y) \in E(G)$, then, $(x, y) \in E\left(I_{3}\right)$, where $x, y \in \mathcal{B}_{1} \cup$ $\mathcal{C} \cup \mathcal{P}_{2 e} \cup \mathcal{P}_{2 i} \cup \mathcal{R}$. For any $x \in \mathcal{P}_{2 i}$, from (43 44), $r\left(x, I_{3}\right)=n+1$ and using Claim 3, we infer that $I_{3}\left[\mathcal{C} \cup \mathcal{P}_{2 e} \cup \mathcal{P}_{2 i} \cup \mathcal{R}\right]$ is a clique. For any $x \in \mathcal{B}_{1}$, from (42), $r\left(x, I_{3}\right)=n$ and again by using Claim 3 , we observe that $I_{3}\left[\mathcal{C} \cup \mathcal{P}_{2 e} \cup \mathcal{B}_{1} \cup \mathcal{R}\right]$ is a clique. Therefore, the only case we have to consider is $x \in \mathcal{P}_{2 i}$ and $y \in \mathcal{B}_{1}$. However, from Table 1 (row 6), this case is not possible. Hence proved.

Lemma 26. $I_{3}[V \backslash \mathcal{A}]$ is a supergraph of $G[V \backslash \mathcal{A}]$. 
Proof. Let $x, y \in V \backslash \mathcal{A}$ be two adjacent vertices in $G$. Note that $V \backslash \mathcal{A}=\left(\mathcal{B}_{2} \cup \mathcal{P}_{e} \cup \mathcal{N}\right) \cup\left(\mathcal{B}_{1} \cup \mathcal{C} \cup \mathcal{P}_{2 e} \cup \mathcal{P}_{2 i} \cup \mathcal{R}\right)$. If $x, y \in \mathcal{B}_{2} \cup \mathcal{P}_{e} \cup \mathcal{N}$, by Observation 26, $x$ and $y$ are adjacent in $I_{3}$. If $x, y \in \mathcal{B}_{1} \cup \mathcal{C} \cup \mathcal{P}_{2 e} \cup \mathcal{P}_{2 i} \cup \mathcal{R}$, then by Lemma 25], $x$ and $y$ are adjacent in $I_{3}$. The remaining case is $x \in \mathcal{B}_{2} \cup \mathcal{P}_{e} \cup \mathcal{N}$ and $y \in \mathcal{B}_{1} \cup \mathcal{C} \cup \mathcal{P}_{2 e} \cup \mathcal{P}_{2 i} \cup \mathcal{R}$.

Let $x \in \mathcal{B}_{2}$. From Table 1 (row 6 ) we note that $y \notin \mathcal{C} \cup \mathcal{P}_{2 e} \cup \mathcal{P}_{2 i}$. Therefore, $y \in \mathcal{B}_{1} \cup \mathcal{R}$. If $y \in \mathcal{B}_{1}$, by (42), $r\left(y, I_{3}\right)=n$ and if $y \in \mathcal{R}$, by (45), $l\left(y, I_{3}\right)=n$. By Observation 2513] $r\left(x, I_{3}\right)=n$ and therefore, $x$ is adjacent to $y$ in $I_{3}$.

Let $x \in \mathcal{P}_{e}$. From Table 1 (rows $6,4,8$ and 5 respectively) $y \notin \mathcal{B}_{1} \cup \mathcal{C} \cup \mathcal{P}_{2 i} \cup \mathcal{R}$. Therefore, $y \in \mathcal{P}_{2 e}$. By (41), $r\left(y, I_{3}\right)=n+1$ and by Observation 2514 $r\left(x, I_{3}\right)=n+1$. Hence, $x$ is adjacent to $y$ in $I_{3}$.

Let $x \in \mathcal{N}$. From Table 1 (row 2), $y \notin \mathcal{R}$. This implies that $y \in \mathcal{B}_{1} \cup \mathcal{C} \cup \mathcal{P}_{2 e} \cup \mathcal{P}_{2 i}$. From Table 2 (rows 2,3 and 4), $x$ is the unique neighbor of $y$ in $\mathcal{N}$. From (41), (42) and (44), $l\left(y, I_{3}\right)=r\left(x, I_{3}\right)$. Hence, $x$ is adjacent to $y$ in $I_{3}$.

Lemma 27. If $x \in \mathcal{N}$ and $y \in V \backslash \mathcal{A}$ such that $(x, y) \notin E(G)$, then, $(x, y) \notin E\left(I_{1} \cap I_{3}\right)$.

Proof. If $y \in \mathcal{B}_{2} \cup \mathcal{P}_{e} \cup \mathcal{N}$, then by Observation 27, $(x, y) \notin E\left(I_{1} \cap I_{3}\right)$. If $y \in \mathcal{R}$, then by (45), $l\left(y, I_{3}\right)=n$. If $y \in \mathcal{P}_{2 i}$ and is not adjacent to any vertex in $\mathcal{N}$, then by [43), $l\left(y, I_{3}\right)=n+1$. By Observation 25ा1] $r\left(x, I_{3}\right)<n$ and therefore, in both the cases, $(x, y) \notin E\left(I_{3}\right)$. Now we consider the remaining cases $(1) y \in \mathcal{P}_{2 i}$ such that $y$ has a neighbor in $\mathcal{N}$ and (2) $y \in \mathcal{B}_{1} \cup \mathcal{C} \cup \mathcal{P}_{2 e}$. From Table 2 (rows 4, 2 and 3 respectively), in each case, $y$ has exactly one neighbor in $\mathcal{N}$ and let this vertex be $z$. Since $x \neq z$, either $\Pi_{1}(x)<\Pi_{1}(z)$ or $\Pi_{1}(z)<\Pi_{1}(x)$.

Suppose $\Pi_{1}(x)<\Pi_{1}(z)$. In $I_{3}$, from the interval assignments for vertices of $\mathcal{C} \cup \mathcal{P}_{2 e}, \mathcal{B}_{1}$ and $\mathcal{P}_{2 i}$ in (41), (42) and (44) respectively, $l\left(y, I_{3}\right)=r\left(z, I_{3}\right)$. From Observation 2511 $r\left(x, I_{3}\right) \leq \Pi_{1}(x)+1<\Pi_{1}(z)+0.5 \leq r\left(z, I_{3}\right)$. Hence, $(x, y) \notin E\left(I_{3}\right)$.

Suppose $\Pi_{1}(x)>\Pi_{1}(z)$. In $I_{1}$, from the interval assignments for vertices of $\mathcal{B}_{1}, \mathcal{P}_{2 e}$ and $\mathcal{P}_{2 i}$ in (15), (16) and (19) respectively, $r\left(y, I_{1}\right)=l\left(z, I_{1}\right)$. For $\mathcal{C}$ in (20), $r\left(y, I_{1}\right) \leq l\left(z, I_{1}\right)+0.5$. From Observation 13/4, $l\left(x, I_{1}\right) \geq l\left(z, I_{1}\right)+1$ and therefore, $(x, y) \notin E\left(I_{1}\right)$. Hence proved.

Lemma 28. Let $x \in \mathcal{N}$ and $y \in V \backslash \mathcal{A}$ such that $(x, y) \in E(G)$. Then, for some $I \in\left\{I_{1}, I_{3}\right\}$, either $l(x, I)=r(y, I)$ or $l(y, I)=r(x, I)$.

Proof. Note that $V \backslash \mathcal{A}=\left(\mathcal{B}_{2} \cup \mathcal{P}_{e} \cup \mathcal{N}\right) \cup\left(\mathcal{B}_{1} \cup \mathcal{C} \cup \mathcal{P}_{2 e} \cup \mathcal{P}_{2 i} \cup \mathcal{R}\right)$. Suppose $y \in \mathcal{B}_{2} \cup \mathcal{P}_{e} \cup \mathcal{N}$. Since $x$ is adjacent to $y$, they belong to the same component in $G\left[\mathcal{B}_{2} \cup \mathcal{P}_{e} \cup \mathcal{N}\right]$, which is either a Type 1 cycle, Type 2 cycle or a path. Let this component be $S=s_{1} s_{2} \ldots s_{t}$, where $\Pi_{1}\left(s_{i+1}\right)=\Pi_{1}\left(s_{i}\right)+1,1 \leq i<t$. Suppose $S$ is a Type 1 cycle. If neither $x$ nor $y$ is $c_{1}$, then by Observation [1011, this condition is satisfied in $I_{1}$. If $x$ or $y$ is $c_{1}$, then by Observation 2222, this is satisfied in $I_{3}$. Suppose $S$ is a Type 2 cycle. If $x$ or $y$ is $c_{1}$, say $x=c_{1}$, then by Observation 232, $r\left(x, I_{3}\right)=l\left(y, I_{3}\right)$. Now we will assume that neither $x$ nor $y$ is $c_{1}$. Suppose $\Pi_{1}(x)>\Pi_{1}(y)$. By Observation 112, $l\left(x, I_{1}\right)=r\left(y, I_{1}\right)$. Moreover, since $c_{t} \in \mathcal{N}$, this holds for the case $x=c_{t}$ and $y=c_{t-1}$ too. Therefore, we can assume that $x, y \notin\left\{c_{1}, c_{t}\right\}$. If $\Pi_{1}(x)<\Pi_{1}(y)$, then, by Observation 2313 $l\left(y, I_{3}\right)=r\left(x, I_{3}\right)$. Finally, suppose $S$ is a path. If $y \in \mathcal{N}$, then, without loss of generality we can assume that $\Pi_{1}(x)<\Pi_{1}(y)$ and therefore, by Observation 242, the condition is satisfied in $I_{3}$. If $y \in \mathcal{B}_{2} \cup \mathcal{P}_{e}$ and $\Pi_{1}(x)<\Pi_{1}(y)$, then, again by Observation [24]2, $r\left(x, I_{3}\right)=l\left(y, I_{3}\right)$ and if $\Pi_{1}(x)>\Pi_{1}(y)$, by Observation $1222 r\left(y, I_{3}\right)=l\left(x, I_{3}\right)$.

Suppose $y \in \mathcal{B}_{1} \cup \mathcal{C} \cup \mathcal{P}_{2 e} \cup \mathcal{P}_{2 i}$. By Table 2 (rows 2, 3 and 4), $x$ is the unique neighbor of $y$ in $\mathcal{N}$. From (41), (42) and (44), it follows that $l\left(y, I_{3}\right)=r\left(x, I_{3}\right)$. By Table1( 1 (row 2), $x$ is not adjacent to any vertex in $\mathcal{R}$. Thus, we have covered all possible cases for $y \in V \backslash \mathcal{A}$. Hence, proved.

Observation 28. If $x \in \mathcal{R}$ and $y \in \mathcal{B}$ are adjacent in $G$, then, $l\left(x, I_{3}\right)=r\left(y, I_{3}\right)=n$. This follows from the fact that $\mathcal{B}=\mathcal{B}_{1} \cup \mathcal{B}_{2}$, 42), 45) and Observation 2513,

\subsubsection{Vertices of $\mathcal{A}$}

Every vertex is assigned the following interval:

$$
\forall v \in \mathcal{A}, f\left(v, I_{3}\right)=[1, n+1] .
$$


Lemma 29. $I_{3}$ is a supergraph of $G$.

Proof. By Lemma 26, $I_{3}[V \backslash \mathcal{A}]$ is a supergraph of $G[V \backslash \mathcal{A}]$. From the interval assignments in $I_{3}$ for vertices in $V \backslash \mathcal{A}$, it is easy to infer that if $x \in V \backslash \mathcal{A}$, then, $f\left(x, I_{3}\right) \subset[1, n+1]$. Since by (46), for any $y \in \mathcal{A}$, $f\left(y, I_{3}\right)=[1, n+1]$, it follows that $y$ is adjacent to $x$. Clearly, $I_{3}[\mathcal{A}]$ is a clique. Therefore, $I_{3}$ is a supergraph of $G$.

\subsection{Proof of $E(G)=E\left(I_{1}\right) \cap E\left(I_{2}\right) \cap E\left(I_{3}\right)$}

We will prove Theorem 11 by showing that $E(G)=E\left(I_{1}\right) \cap E\left(I_{2}\right) \cap E\left(I_{3}\right)$. We have already proved that $I_{1}$, $I_{2}$ and $I_{3}$ are supergraphs of $G$ (in Lemmas 13, 21] and 29, respectively). In this section, we will show the following: If two vertices $s$ and $t$ are not adjacent in $G$, then there exists at least one interval graph $I \in$ $\left\{I_{1}, I_{2}, I_{3}\right\}$, such that $(s, t) \notin I$. Recall the partitioning of $V$ illustrated in Figure 2. $V=\mathcal{A} \cup \mathcal{N} \cup \mathcal{C} \cup \mathcal{R} \cup \mathcal{B} \cup \mathcal{P}$. Now we will consider one by one all possible cases and in each case show that $s$ and $t$ are not adjacent in at least one of the interval graphs.

$s \in \mathcal{A}, \boldsymbol{t} \in \boldsymbol{V}:$ If $t \in \mathcal{A}$, then by Lemma 8, $(s, t) \notin E\left(I_{1}\right)$. If $t \in \mathcal{N}$, then by Lemma 16, $(s, t) \notin E\left(I_{1} \cap I_{2}\right)$ and if $t \in V \backslash(\mathcal{A} \cup \mathcal{N})$, then by Lemma $14(s, t) \notin E\left(I_{1}\right)$.

$s \in \mathcal{N}, \boldsymbol{t} \in \boldsymbol{V} \backslash \mathcal{A}:$ By Lemma 27, $(s, t) \notin E\left(I_{1} \cap I_{3}\right)$.

$s \in \mathcal{C}, t \in \boldsymbol{V} \backslash(\mathcal{A} \cup \mathcal{N})=\mathcal{C} \cup \mathcal{P} \cup \mathcal{R} \cup \mathcal{B}:$ If $t \in \mathcal{C} \cup \mathcal{P}$, by Lemma 18, $(s, t) \notin E\left(I_{1} \cap I_{2}\right)$. If $t \in \mathcal{R} \cup \mathcal{B}$, by Observation 2112, $(s, t) \notin E\left(I_{2}\right)$.

$s \in \mathcal{P}, t \in \boldsymbol{V} \backslash(\mathcal{A} \cup \mathcal{N} \cup \mathcal{C})=\mathcal{P} \cup \mathcal{R} \cup \mathcal{B}$ : If $t \in \mathcal{P}$, by Lemma 18, $s$ and $t$ are not adjacent in either $I_{1}$ or $I_{2}$. Let $t \in \mathcal{R} \cup \mathcal{B}$. If $s \in \mathcal{P}_{e} \cup \mathcal{P}_{2 e}$, then by Observation 212. $(s, t) \notin E\left(I_{2}\right)$. Finally, let $s \in \mathcal{P}_{2 i}$. If $t \in \mathcal{R}$, then by Observation 2111 $(s, t) \notin E\left(I_{2}\right)$ and if $t \in \mathcal{B}$, by Lemma 24, $(s, t) \notin E\left(I_{2} \cap I_{3}\right)$.

$s, t \in \mathcal{R} \cup \mathcal{B}:$ By Lemma 20 (Statement 2), $(s, t) \notin E\left(I_{2}\right)$.

\subsection{Proof of Theorem 1}

We have proved that $G$ has a 3-box representation. Now we will show that in this 3-box representation, any two intersecting boxes intersect only at their boundaries and hence complete the proof of Theorem 1. For this to happen, the following condition needs to be satisfied:

Condition 1. Let $s$ and $t$ be adjacent in $G$. For some $I \in\left\{I_{1}, I_{2}, I_{3}\right\}$, either $l(s, I)=r(t, I)$ or $l(t, I)=$ $r(s, I)$.

As in the previous section, we will consider one by one all the possible cases:

$s \in \mathcal{A}, t \in \boldsymbol{V}:$ If $t \in \mathcal{A}$, then by Observation 8 and if $t \in \mathcal{N}$, by Observation 136. Condition 1 is satisfied. By Table 1 (rows 1-3), $s$ is not adjacent to any vertex in $V \backslash(\mathcal{A} \cup \mathcal{N})$.

$s \in \mathcal{N}, t \in V \backslash \mathcal{A}$ : By Lemma 28, Condition 1 is satisfied.

$s \in \mathcal{C}, t \in \boldsymbol{V} \backslash(\mathcal{A} \cup \mathcal{N})=\mathcal{C} \cup \mathcal{P} \cup \mathcal{R} \cup \mathcal{B}$ : By Table 1 (rows 4-6), $t \notin \mathcal{P} \cup \mathcal{R} \cup \mathcal{B}$. Therefore, the only case to be considered is $t \in \mathcal{C}$. Let $s, t \in C$, where $C \subseteq \mathcal{C}$ is a special cycle. Let $C=c_{1} c_{2} \ldots c_{t}$ where $c_{1}$ is the special vertex. If $x=c_{1}$, then by Observation 1511. Condition 1 is satisfied. If neither $x$ nor $y$ is $c_{1}$, then by Observation 1911. Condition 1 is satisfied in $I_{2}$.

$s \in \mathcal{P}, \boldsymbol{t} \in \boldsymbol{V} \backslash(\mathcal{A} \cup \mathcal{N} \cup \mathcal{C})=\mathcal{P} \cup \mathcal{R} \cup \mathcal{B}$ : If $t \in \mathcal{P}$, then by Observation 18, Condition 1 is met in $I_{2}$. If $t \in \mathcal{R}$, then from Table 1 (row 5) we infer that $s \in \mathcal{P}_{2 i}$. By Observation 14. Condition 1 is met in $I_{1}$. By Table 1 (row 6$), t \notin \mathcal{B}$. 
$s \in \mathcal{R}, \boldsymbol{t} \in \boldsymbol{V} \backslash(\mathcal{A} \cup \mathcal{N} \cup \mathcal{C} \cup \mathcal{P})=\mathcal{R} \cup \mathcal{B}:$ By Table 1 (row 10) $t \notin \mathcal{R}$. By Observation 28, Condition 1 , is satisfied in $I_{3}$.

$s, t \in \mathcal{B}:$ Condition 1 is satisfied in $I_{2}$ (See Figure 3).

Thus, we have completed the proof for cubic graphs. Now, applying Lemma 2, it follows that any graph with maximum degree 3 has a 3-box representation. Thus, we have proved Theorem 1 .

\section{Algorithmic aspects}

Now we briefly explain how our construction of the 3-box representation can be realized in $O(n)$ time, where $n$ is the number of vertices in the graph. Firstly, we note that the process can be split into three stages: (1) Partitioning the vertex set as illustrated in Figure 2. (2) ordering the vertices of $\mathcal{A}, \mathcal{B}_{2} \cup \mathcal{P}_{e} \cup \mathcal{N}$ and $\mathcal{C} \cup \mathcal{P}$ according to Definitions 7, 8 and 10 and finally, (3) assigning intervals to all the vertices. In Stage (1) the first step is to extract special cycles and special paths as described in Algorithm 1. This is the only non-trivial part of the construction and we analyze its complexity in the appendix. We will show that a special cycle or path can be extracted from a graph with maximum degree 3 in time linear to the number of vertices in it. This will imply that Algorithm 1 can be implemented in $O(n)$ time. Algorithm 2 takes $O(n)$ time since in every iteration we need to only check if a vertex in $\mathcal{N}_{1}$ has two neighbors in $\mathcal{A}_{1}$ in that iteration and accordingly move or retain the vertex and its neighbors. It is easy to see that the finer partitioning of $\mathcal{P}, \mathcal{N}$ and $\mathcal{B}$ can be accomplished in linear time. Stage (2) involves ordering the vertices of sets $\mathcal{A}, \mathcal{B}_{2} \cup \mathcal{P}_{e} \cup \mathcal{N}$ and $\mathcal{C} \cup \mathcal{P}$ component wise. Since each of these sets induce a graph of maximum degree 2, they can be ordered in linear time. Stage (3) only involves assignment of intervals to the vertices and can be achieved in linear time.

\section{Conclusion}

We showed that every graph of maximum degree 3 has a 3-box representation and therefore, its boxicity is at most 3. One interesting question is whether we can characterize cubic graphs which have a 2-box representation. Answering this will also determine if the boxicity of a cubic graph can be computed in polynomial time. One could also try to extend the proof techniques used in this paper to graphs with maximum degree 4 and 5 in order to improve the bounds on their boxicity.

\section{References}

1. A. Adiga, D. Bhowmick, L. S. Chandran, Boxicity and poset dimension., in: M. T. Thai, S. Sahni (eds.), COCOON, vol. 6196 of Lecture Notes in Computer Science, Springer, 2010.

2. L. S. Chandran, M. C. Francis, N. Sivadasan, Boxicity and maximum degree, J. Comb. Theory Ser. B 98 (2) (2008) 443-445.

3. L. S. Chandran, N. Sivadasan, Boxicity and treewidth, J. Comb. Theory Ser. B 97 (5) (2007) 733-744.

4. M. B. Cozzens, Higher and multi-dimensional analogues of interval graphs, Ph.D. thesis, Department of Mathematics, Rutgers University, New Brunswick, NJ (1981).

5. M. B. Cozzens, F. S. Roberts, Computing the boxicity of a graph by covering its complement by cointerval graphs, Disc. Appl. Math. 6 (1983) 217-228.

6. L. Esperet, Boxicity of graphs with bounded degree, European J. Combin. 30 (5) (2009) 1277-1280.

7. S. Felsner, M. C. Francis, Contact representations of planar graphs with cubes, in: Proc. 27th Annu. ACM Sympos. Comput. Geom. (SoCG 2011), 2011.

8. J. Kratochvíl, A special planar satisfiability problem and a consequence of its NP-completeness, Disc. Appl. Math. 52 (1994) 233-252.

9. F. S. Roberts, Recent Progresses in Combinatorics, chap. On the boxicity and cubicity of a graph, Academic Press, New York, 1969, pp. 301-310.

10. E. R. Scheinerman, Intersection classes and multiple intersection parameters, Ph.D. thesis, Princeton University (1984).

11. E. R. Scheinerman, D. B. West, The interval number of a planar graph: Three intervals suffice, J. Comb. Theory Ser. B 35 (1983) 224-239.

12. C. Thomassen, Interval representations of planar graphs, J. Comb. Theory Ser. B 40 (1986) 9-20.

13. M. Yannakakis, The complexity of the partial order dimension problem, SIAM J. Alg. Disc. Math. 3 (3) (1982) 351-358. 


\section{A Appendix}

Let $G$ be a graph with maximum degree 3 .

\section{A.1 Extending a non-special induced cycle or path}

Suppose $C$ is a non-special induced cycle in $G$. Then, by Definition 10 it follows that there exists a vertex $x \in C$, such that $C \backslash\{x\}$ belongs to a cycle or path of length $|C|+1$. We call such a vertex a removable vertex. Extending a non-special induced cycle $C$ corresponds to removing a removable vertex $x$ and adding two new vertices $a$ and $b$ such that $(C \backslash\{x\}) \cup\{a, b\}$ is an induced cycle or a path. There are only two possible ways in which a non-special induced cycle can be extended and these are illustrated in Figure 4 The vertices which are added to or removed from $C$ in an extension operation are called participating vertices. In the figure, $x, a$ and $b$ are the participating vertices.
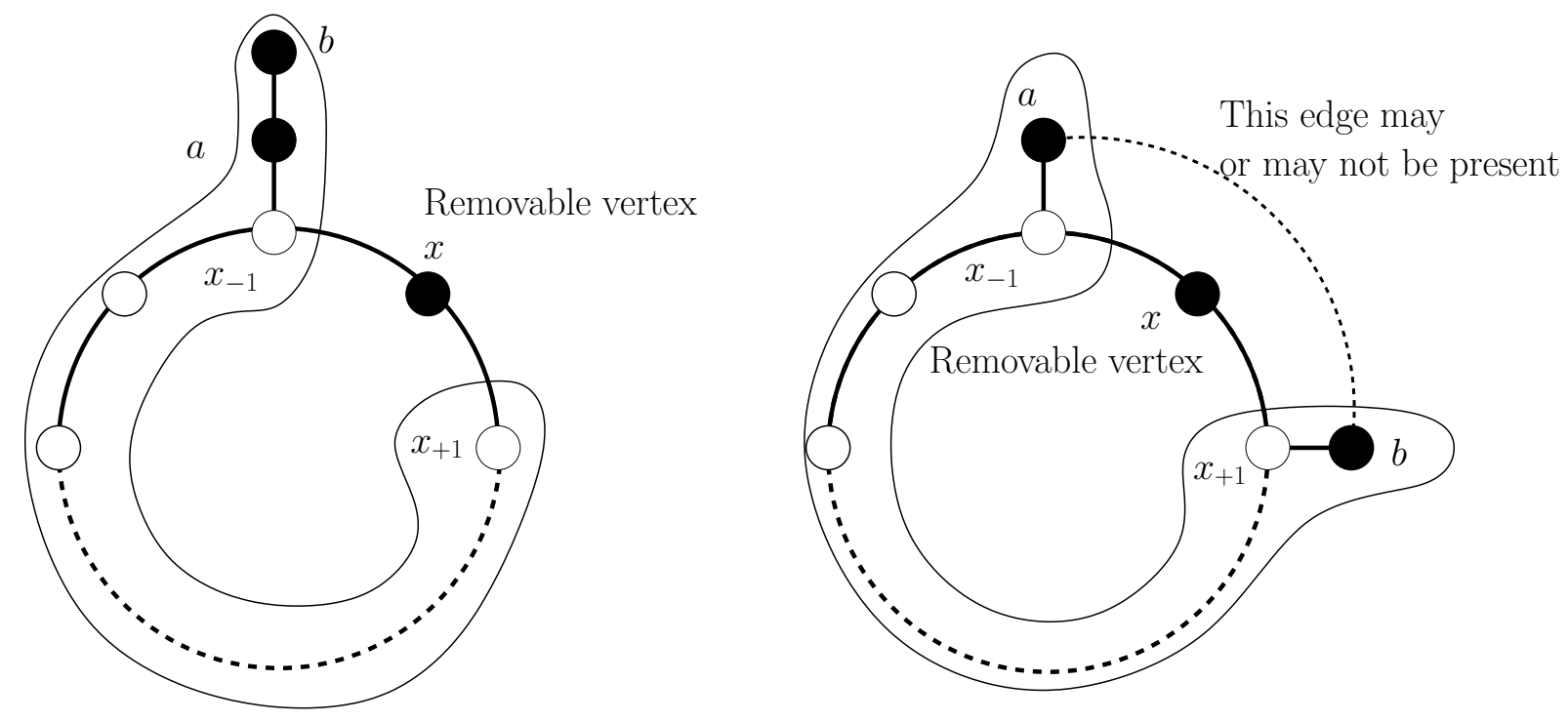

(a)

(b)

Fig. 4. The possible ways in which a non-special induced cycle can be extended. The vertices marked black are the participating vertices.

Lemma 30. Suppose $C$ is a non-special induced cycle and $x \in C$. It takes constant time to verify whether $x$ is a removable vertex or not. If $x$ is a removable vertex, then, the extension of $C$ by removing $x$ can again be achieved in constant time.

Proof. Consider the possible ways in which $C$ can be extended as shown in Figure 4. To verify if $x$ is a removable vertex, we need to only check if the vertices $a$ and $b$ exist. Similarly, given that $x$ is a removable vertex, we need to only find $a$ and $b$ to extend $C$ by removing $x$. Recalling that $\Delta(G) \leq 3$, it is easy to see that this can be done in constant time.

Let $P$ be a non-special induced path in $G$. By Definition 2, it implies that either:

1. it is not maximal in the sense that it is part of an induced cycle or a longer induced path, or

2. for some end point of $P$, say $x, P \backslash\{x\}$ belongs to an induced cycle of size $\geq|P|$ or an induced path of length $\geq|P|+1$. We call $x$ a removable end point of $P$. 
Extending a non-special path $P$ corresponds to the following operations:

1. If $P$ is not maximal, then, we add a new vertex $y$ to $P$ such that $P \cup\{y\}$ is an induced path or cycle. Clearly, $y$ must be a neighbor of an end point of $P$ such that it is not adjacent to any interior vertex of $P$.

2. If $P$ is maximal, then, we remove a removable end point $x$ and either

(a) add a single new vertex $a$ such that $(P \backslash\{x\}) \cup\{a\}$ is an induced cycle (note that in this case $a$ has to be adjacent to the neighbor of $x$ in $P$ ) OR

(b) add two new vertices $a$ and $b$ such that $(P \backslash\{x\}) \cup\{a, b\}$ is an induced cycle or a path (in this case, $a$ and $b$ are adjacent and $a$ is adjacent to the neighbor of $x$ in $P$ ).

This is illustrated in Figure 5

As in the case of extending a cycle, the vertices which are added to or removed from $P$ are called participating vertices. In case $1, y$ is the participating vertex. In case 2(a), $x$ and $a$ are participating vertices and in case $2(\mathrm{~b}), x, a$ and $b$ are participating vertices.

Removable end point

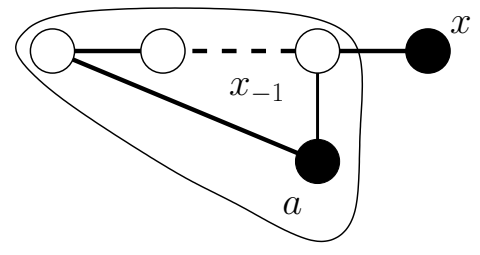

(a)

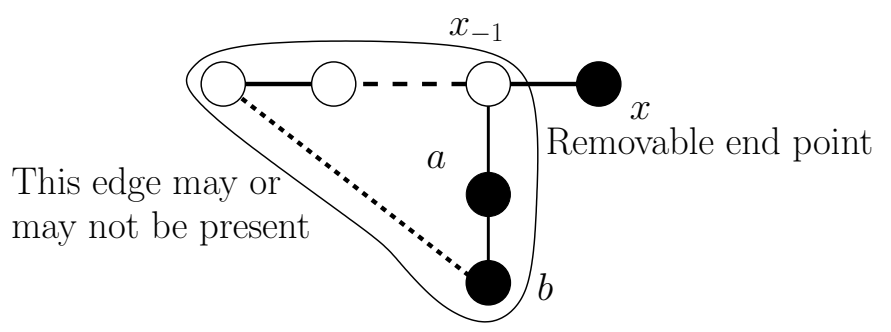

(b)

Fig. 5. The ways in which a non-special induced path can be extended. The vertices marked black are the participating vertices.

Lemma 31. If $P$ is an induced path, then, in constant time, it can be verified whether it is a special path or not. If not, then, in constant time it can be extended.

Proof. First we need to check if $P$ is maximal or not, that is, whether it is part of a larger induced cycle or a longer induced path. This can be done in constant time. If $P$ is maximal, then, we need to check if there is a removable end point and then extend $P$ by removing it. For this, as shown in Figure 5 , we need to check if the vertices $x, a$ and $b$ exist. Since $\Delta(G) \leq 3$, it is easy to see that this can be done in constant time. It is also trivial to verify that the extension can be achieved in constant time. Hence proved.

\section{A.2 An algorithm to find a special cycle or path}

We now give an iterative algorithm to obtain a special cycle or path. The outline of the algorithm is as follows: Let $S$ be the set which holds the vertices of the special cycle or path at the termination of the algorithm. We start with $S$ containing an arbitrary vertex. In each iteration, we extend it as described in Section A.1. The algorithm terminates when $S$ induces a special cycle or path. In Algorithm 3, we present an outline of this procedure.

\section{A.2.1 Potential removable vertices}

From Lemma 31, we note that in constant time we can recognize a non-special induced path and extend it. However, in view of Lemma 30, to recognize and extend a non-special induced cycle in constant time, we first need a strategy to find a removable vertex. For efficiently finding removable vertices, we maintain a list of potential removable vertices which is updated in each iteration. 


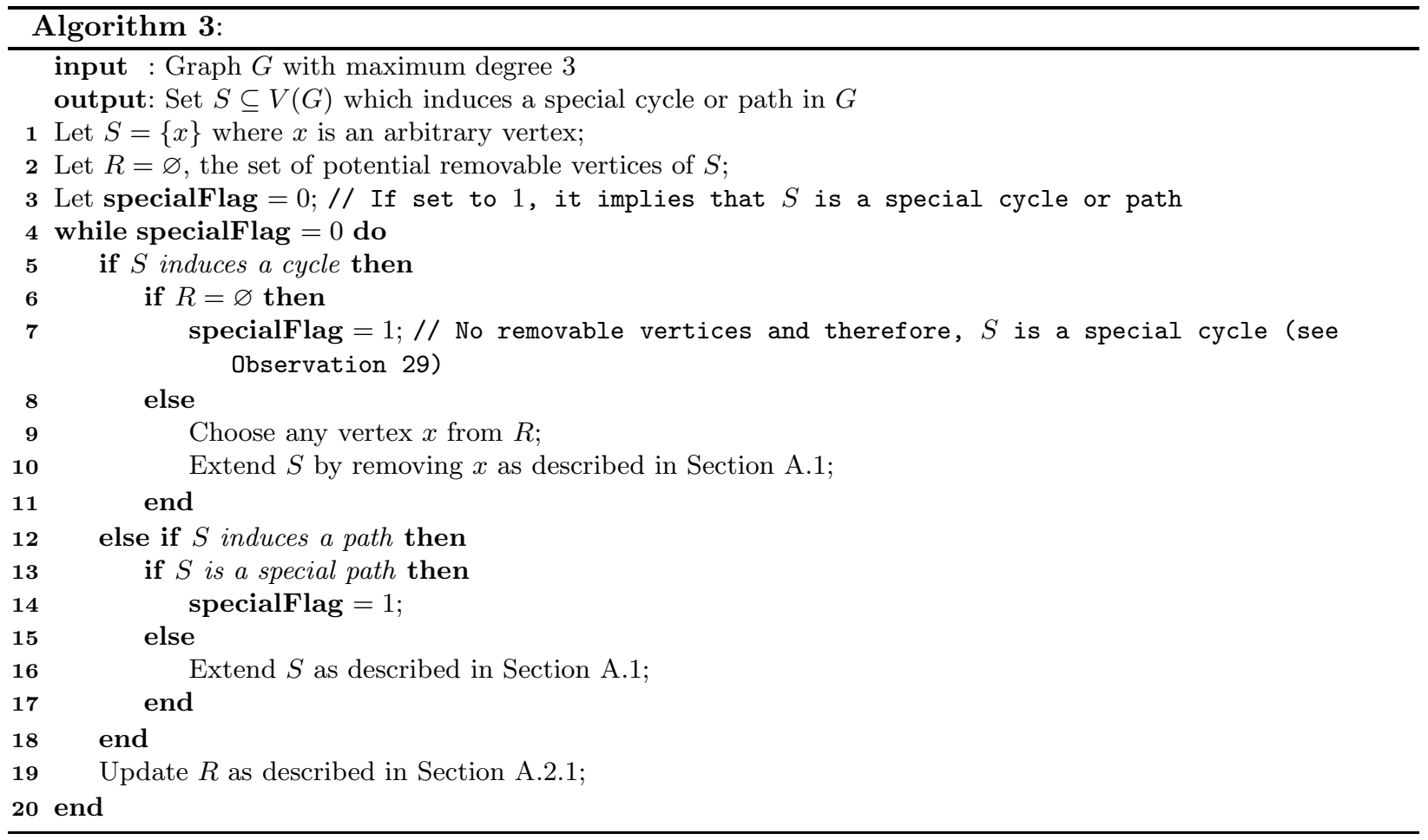

Definition 12. Potential removable vertices: $A$ vertex $x \in S$ is a potential removable vertex if it has two neighbors in $S$, say $x_{-1}$ and $x_{+1}$ and satisfies at least one of the following conditions: There are two vertices $a$ and $b$ such that

1. $a$ is adjacent to only $x_{-1}$ (or $x_{+1}$ ) in $S \backslash\{x\}$ and $b$ is adjacent to a and not any vertex in $S \backslash\{x\}$. This corresponds to case (a) in Figure 4; $O R$

2. $a$ is adjacent to $x_{-1}$ in $S \backslash\{x\}$ and $b$ is adjacent to only $x_{+1}$ in $S \backslash\{x\}$. This corresponds to case (b) in Figure 4.

From the definition of removable vertices at the beginning of Section A.1, we infer the following:

Observation 29. If $S$ induces a cycle, then, all potential removable vertices in $S$ will correspond to removable vertices. If there are no potential removable vertices, then, it implies that $S$ is a special cycle.

Lemma 32. In constant time, we can check if a particular vertex in $S$ is a potential removable vertex.

The proof is similar to that of Lemma 30.

In the algorithm, we maintain a set of all potential removable vertices, which we denote as $R$. From Observation 29, it follows that if $S$ induces a cycle, we can decide whether it is special or not by just checking if $R$ is empty or not. Therefore, given an induced cycle and the corresponding $R$, we can recognize in constant time whether it is a special cycle or not. Now, we show that after each extension of $S, R$ can be updated in constant time.

Lemma 33. In each iteration of Algorithm [3, the set of potential removable vertices, $R$ can be updated in constant time.

Proof. Let us consider a vertex $x \in R$ before extension of $S$. Let $X$ denote the set containing $x$ and the associated vertices $x_{-1}, x_{+1}, a$ and $b$ (see Definition 12). Since $S$ is extended, there are participating vertices. We observe that $x$ will remain as a potential removable vertex if no vertex in $X$ and no neighbor of $X$ is a participating vertex. This implies that if a vertex is at a distance 5 or more from any participating vertices, then, clearly its status as a potential removable vertex or not remains unchanged. Therefore, we need to 
only check all the vertices at a distance 4 or less from each participating vertex. The number of such vertices is a constant since $\Delta(G)=3$ and by Lemma 32 verifying for each vertex takes only constant time. Hence proved.

Lemma 34. If the special cycle or path extracted by Algorithm 3 is of size $l$, then, the total number of iterations required is at most $2 l+2$.

Proof. We will prove the lemma by showing that in Algorithm 3, for every two iterations (excluding the last two) the size of $S$ increases by at least 1 . If $S$ induces a cycle at the beginning of the $i$ th iteration, then from Section A.1 it follows that $S$ is extended to a cycle or path of size $|S|+1$ at the end of the iteration. If $S$ induces a path at the beginning of the $i$ th iteration, either $S$ is extended to a cycle or path of size $|S|+1$ or to a cycle of size $|S|$ at the end of the iteration. In the latter case, assuming $S$ is not a special cycle in the $(i+1)$ th iteration, it is extended to a cycle or path of size $|S|+1$ at the end of the $(i+1)$ th iteration. Hence, proved.

From Lemmas 30 33, it follows that Lines 10, 16 and 19 in Algorithm 3 require constant time. The sets $S$ and $R$ may be implemented as doubly linked lists and with each vertex we can associate membership flags and pointers to its place in each of the linked lists. Given this setup, each iteration requires constant time. From Lemma 34 the number of iterations is bounded by $2 l+2$. Therefore, the algorithm takes $O(l)$ time to terminate. Since the total number of vertices in the set of special cycles and paths will be bounded above by $n$, the overall running time of Algorithm 1 is $O(n)$. 